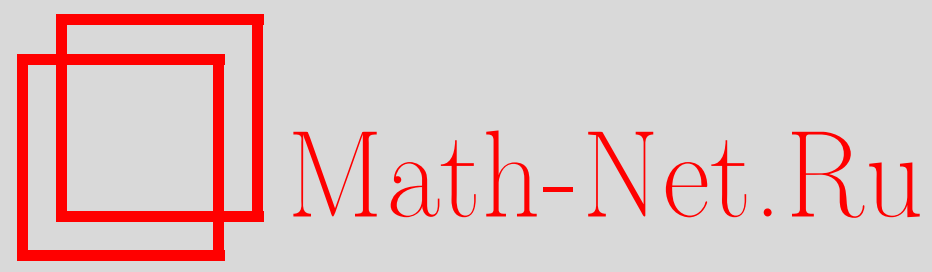

Г. В. Калачев, Порядок мощности плоских схем, реализующих булевы функции, Дискрет. матем., 2014, том 26, выпуск $1,49-74$

DOI: https://doi.org/10.4213/dm1267

Использование Общероссийского математического портала Math-Net.Ru подразумевает, что вы прочитали и согласны с пользовательским соглашением http://www.mathnet.ru/rus/agreement

Параметры загрузки:

IP: 54.84 .234 .179

26 апреля 2023 г., 12:51:25 


\title{
Порядок мощности плоских схем, реализующих булевы функции
}

\author{
(ㄷ) 2014 г. Г. В. Калачев ${ }^{1}$
}

\begin{abstract}
В работе исследуется функция Шеннона "мощности" плоских схем, реализующих булевы функции. Рассматриваются две меры мощности. Первая мера - потенциал, он равен количеству выходов элементов, выдающих единицу на заданном входном наборе схемы. Вторая мера задается на паре наборов и равна количеству выходов элементов, значения на которых различны на этих двух наборах. Для обеих мер мощности рассматриваются как среднее значение, так и максимальное по всевозможным входным наборам. Получен порядок функции Шеннона мощности схем и, в частности, показано, что для рассматриваемых мер мощности порядок функции Шеннона один и тот же и равен $2^{n / 2}$.
\end{abstract}

Ключевые слова: схемы из функциональных элементов, плоские схемы, мощность, порядок функции Шеннона.

\section{1. Введение.}

С. С. Кравцов в статье [1] ввел понятие схемы из клеточных элементов, далее также называемых плоскими схемами, и доказал, что для реализации произвольной булевой функции такой схемой требуется $O\left(2^{n}\right)$ элементов, причем существуют функции, для реализации которых необходимо порядка $2^{n}$ элементов. Более подробно площадь плоских схем исследовал Д. А. Жуков, и он, в частности, в статье [2] получил порядок функции Шеннона для частичных булевых функций $O(|D|)$, где $D$ - область определения. Н.А. Шкаликова в работе [3] исследовала связь между площадью плоских схем и объемом трехмерных схем, реализующих булевы операторы. Она показала, что если оператор реализуется трехмерной схемой с объемом $V$, то его можно реализовать плоской схемой с площадью $O\left(V^{3 / 2}\right)$, причем порядок этой оценки нельзя понизить.

О. М. Касим-Заде исследовал активность схем из функциональных элементов. В работе [4] качестве меры активности схемы он ввел понятие $P$-активности: на каждом входном наборе рассматривается количество элементов, на вход которых подается единица или сам элемент выдает единицу, после этого берется максимум по всем наборам. Касим-Заде показал, что в некотором базисе для любой булевой функции от $n$ переменных можно построить схему, $P$-активность которой имеет порядок $O\left(n^{2}\right)$. Ю. С. Шуткин в работе [5] исследовал мощность контактных схем и получил линейный порядок функции Шеннона.

\footnotetext{
${ }^{1}$ Место работы: МГУ им. М. В. Ломоносова

e-mail: gleb.Kalachev@yandex.ru
} 
В данной работе вводится понятие мощности плоской схемы как модели микросхемы, вычисляющей данную булеву функцию. Плоская схема состоит из логических элементов и соединяющих их проводов. Рассматриваем такие схемы, в которых основная энергия тратится лишь на переключение логических элементов (например сделанные на основе полевых транзисторов) либо такие, что ток через базу транзисторов пренебрежимо мал. В такой модели схем энергия тратится на паразитные емкости, которыми обладают проводники и затворы полевых транзисторов. Емкость провода пропорциональна его длине, поэтому энергия, выделяемая на проводе при переключении, также пропорциональна его длине. Исходя из этих соображений и вводится определение мощности плоской схемы, задаваемой на паре наборов значений входных переменных и определяемой как количество элементов схемы, сменивших свое значение на этой паре наборов. В этой работе показано, что функция Шеннона как для средней, так и для максимальной мощности плоских схем, pеализующих булевы функции, имеет порядок $2^{n / 2}$. Также здесь рассмотрена и другая мера мощности плоских схем, задаваемая на наборе значений входных переменных и равная количеству элементов схемы, принимающих значение 1 на этом наборе, и показано, что ее функция Шеннона имеет тот же порядок.

Заметим, что в отличие от схем из функциональных элементов для площади плоских схем известен лишь порядок функции Шеннона.

Автор выражает глубокую благодарность научному руководителю д.ф.-м.н., профессору Э.Э. Гасанову за постановку задачи и внимание к работе.

\section{2. Определения и формулировка результатов.}

\section{1. Плоские схемы}

Клеточным элементом будем называть булев оператор, у которого в сумме не более четырех входов и выходов, причем каждому его входу и каждому выходу сопоставлена некоторая метка из множества $\{l, r, t, b\}$, причем метки не повторяются.

Метки будем также называть сторонами элемента:

- $l$ - левая сторона;

- $r$ - правая сторона;

$-t$ - верхняя сторона;

$-b$ - нижняя сторона.

Клеточный элемент будем изображать в виде единичного квадрата на плоскости. При этом входам и выходам элемента сопоставляются стороны квадрата в соответствии с присвоенными им метками.

Метки, присвоенные входам (выходам) оператора будем называть входами (въходами) элемента. Метки, не присвоенные ни входам, ни выходам, будем называть изоляторами. Множество входов (выходов) элемента $e$ будем обозначать $i n(e)$ $($ out $(e))$. Входы и выходы элемента будем называть его контактами.

Заметим, что это определение немного отличается от обычного тем, что допускается, чтобы на разных выходах реализовывались разные нетождественные функции.

Если на всех выходах элемента реализуются тождественные функции, то будем называть элемент коммутационным, иначе - логическим. 
Коммутационный элемент соответствует либо проводнику в микросхеме, либо пересечению проводов, либо тождественной функции, служащей для усиления сигнала.
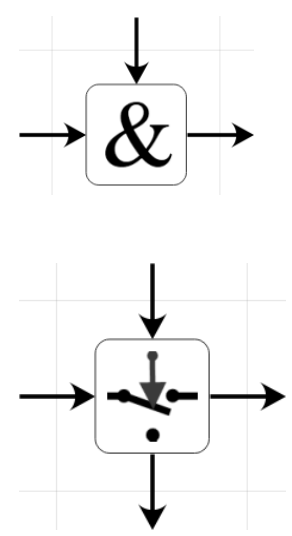

$r=l \bar{t}, b=l t$

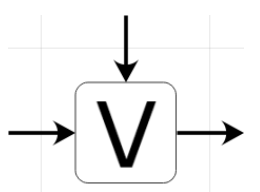

(а) обычные логические элементы

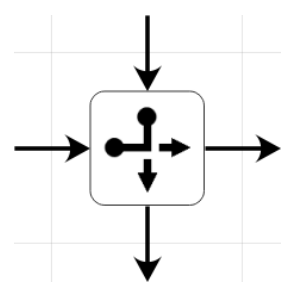

$r=l \bar{t}, b=\bar{l} t$
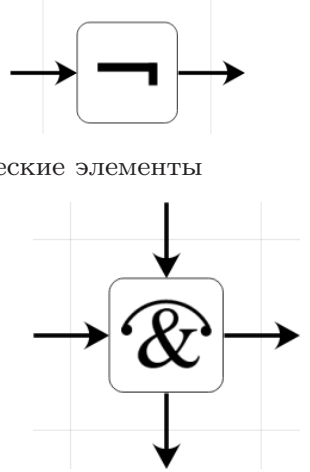

$r=l, b=l t$
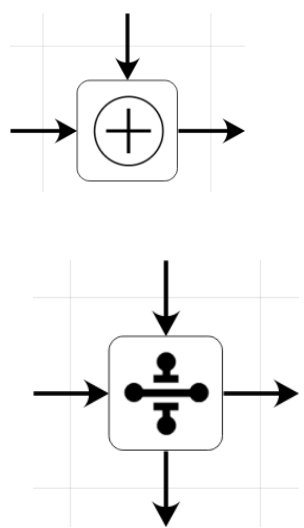

$r=l, b=\bar{l} t$

(b) логические элементы, реализующие две различные функции

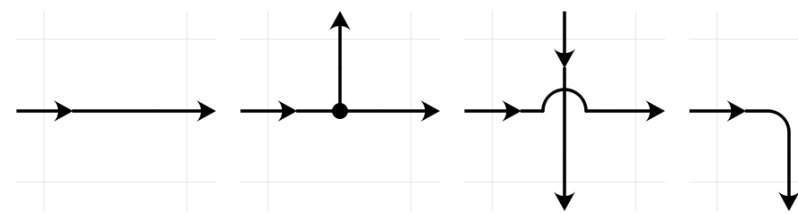

(c) коммутационные элементы

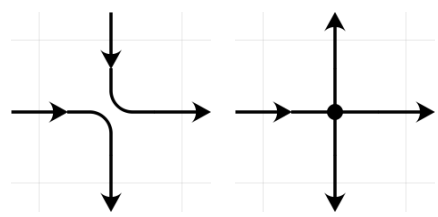

Рис. 1. Примеры клеточных элементов.

Описывать элемент будем уравнениями, которые задают его оператор, заменяя все переменные в них на сопоставленные им метки $(l, r, t$ или $b)$. Тогда в левой части каждого уравнения будет стоять выходная метка, а в правую будут входить только входные метки.

На рис. 1 приведены примеры клеточных элементов.

Для удобства введем пустой клеточный элемент - изолирующий (будем обозначать $\lambda)$.

Всюду далее значок := будет обозначать «по определению равно».

Через $E$ обозначим множество всех клеточных элементов, $N_{E}:=|E|$.

Сетъю из клеточных элементов на множестве $M \subset \mathbb{Z}^{2}$ над множеством $E^{\prime} \subseteq E$ будем называть отображение $K: M \rightarrow E^{\prime}$, при этом $E^{\prime}$ будем называть базисом сети.

Элемент $K(x, y)$ будем называть элементом схемъ $K$ с координатами $(x, y)$. Элемент с приписанными ему координатами будем называть элементом схемы. Левой, правой, верхней и нижней сторонами элемента е с координатами $(x, y)$ будем называть точки с координатами $\left(x-\frac{1}{2}, y\right),\left(x+\frac{1}{2}, y\right),\left(x, y-\frac{1}{2}\right)$ и $\left(x, y+\frac{1}{2}\right)$ соответственно (на рисунках ось $y$ будет направлена вниз).

Будем говорить, что сеть $K$ из клеточных элементов корректна, если для любых двух таких элементов $x$ и $y$ схемы $K$, что сторона $a$ элемента $x$ совпадает со стороной 
$b$ элемента $y$, выполнено одно из условий:

- один из элементов $x, y$ - изолирующий,

- стороны $a$ и $b$ являются изоляторами,

- либо одна из них является входом, другая - выходом, например, $a$ - выход, a $b$ - вход; в таком случае будем говорить, что выход $a$ подключен к входу $b$.

Множество $M$ будем называть носителем сети $K$.

Введем понятие графа корректной сети из клеточных элементов $K$ (будем обозначать его $\left.G_{K}\right)$ : это ориентированный граф, вершинами которого являются входы и выходы элементов схемы. Если выход одного элемента подключен ко входу другого, то им будет соответствовать одна и та же вершина графа (будем говорить, что эта вершина является выходом первого элемента и входом второго). Из вершины $a$ в вершину $b$ ведет ребро в том и только том случае, когда существует такой элемент $e$, что $a$ является его входом, $b$ - выходом, причем функция, реализуемая на выходе $b$, существенно зависит от входа $a$.

Плоской схемой или схемой из клеточных элементов на множестве $M \subset \mathbb{Z}^{2}$ над базисом $E^{\prime} \subseteq E$ будем называть корректную сеть из клеточных элементов, в графе которой нет ориентированных циклов. Множество $M$ будем называть носителем схемы $K$.

Далее везде по умолчанию используем базис $E$, то есть считаем, что у нас есть все клеточные элементы.

Если вход (выход) элемента не подключен к выходу (входу) другого элемента, будем его называть входом (выходом) схемы.

Контактами схемы будем называть ее входы и выходы. Множество входов (выходов) схемы $K$ будем обозначать $\operatorname{In}(K)(\mathrm{Out}(K))$.

Узлами схемы $K$ будем называть вершины графа $G_{K}$.

Если $M$ - носитель схемы $K$, то величину $|M|$, равную количеству элементов множества $M$, будем называть площадъю схемы $K$ и обозначать $|K|$.

Расстоянием между узлами схемы будем называть расстояние между соответствующими вершинами в $G_{K}$. Расстояние от узла $a$ до узла $b$ на схеме $K$ будем обозначать $\rho_{K}(a, b)$.

Подсхемой схемы $K$ с носителем $M_{0} \subseteq M$ будем называть схему $\left.K\right|_{M_{0}}$, получающуюся из $K$ выбрасыванием клеточных элементов, соответствующих множеству $M \backslash M_{0}$. Если схема $K$ фиксирована, то иногда будем говорить просто подсхема $M_{0}$.

Каждой плоской схеме $K$ можно сопоставить схему из функциональных элементов $\operatorname{Circ}(K)$ следующим образом:

1) каждой функции $f_{s, i}$, которую реализует $i$-й выход элемента $s$ клеточной схемы, сопоставим функциональный элемент $e_{s, i}$, реализующий $f_{s, i} ;$ если $i$-й и $j$-й выходы являются выходами одной и той же функции, то им будет соответствовать один и тот же функциональный элемент;

2 ) если $i$-й выход $s_{1}$ подключен к $j$-му входу $s_{2}$, то соединим выход элемента $e_{s_{1}, i}$ с $j$-ми входами элементов $e_{s_{2}, k}$ для всех $k$, для которых $f_{s_{2}, k}$ зависит от $j$-го аргумента; 
3) удалим из схемы все тождественные функции, подсоединив их вход ко всем их выходам.

Будем говорить, что схема $K$ реализует булев оператор $F_{K}$, если схема из функциональных элементов $\operatorname{Circ}(K)$ реализует $F_{K}$.

Назовем схему $K$ минималъной над базисом $E^{\prime} \subseteq E$, если она обладает минимальной площадью среди всех схем над базисом $E^{\prime}$, реализующих $F_{K}$.

Обозначим $S_{E^{\prime}}(F)$ площадь минимальной схемы, реализующей оператор $F$. Если $E^{\prime}=E$, то будем просто писать $S(F)$.

Будем говорить, что плоские схемы $K_{1}$ и $K_{2}$ равны и писать $K_{1}=K_{2}$, если существует параллельный перенос плоскости, который позволяет совместить схемы $K_{1}$ и $K_{2}$, иначе будем говорить, что $K_{1}$ и $K_{2}$ различны.

Замечание 1. Обычно, когда рассматривают плоские схемы, предполагается, что они имеют форму прямоугольника, и входы и выходы расположены по периметру. Но здесь мы не накладываем ограничения на геометрию схемы, поскольку схема, реализующая булеву функцию, может быть частью большой схемы, реализующей булев оператор. А ограничения на геометрию есть лишь для всей схемы устройства, а не для ее составных частей.

\section{2. Меры мощности схем.}

Для каждой схемы $K$ зафиксируем некоторую нумерацию ее узлов. На $i$-м узле реализуется некоторая функция $g_{i}$ от входных переменных схемы $K$ (на входах считаем, что реализуются тождественные функции).

Везде далее будем считать, что схема $K$ имеет $n$ входов, $l$ узлов и $g_{i}-$ функция, реализуемая в $i$-м узле схемы $K$.

Состоянием схемы $K$ на входном наборе $x$ назовем вектор

$$
s_{K}(x):=\left(g_{1}(x), \ldots, g_{l}(x)\right) .
$$

Для $v=\left(v_{1}, \ldots, v_{q}\right) \in\{0,1\}^{q}$ введём обозначение $|v|:=v_{1}+v_{2}+\ldots+v_{q}$.

Введем операцию $\oplus:\{0,1\}^{q} \times\{0,1\}^{q} \rightarrow\{0,1\}^{q}$ - покомпонентное сложение по модулю 2 , то есть $\left(v_{1}, \ldots, v_{q}\right) \oplus\left(u_{1}, \ldots, u_{q}\right):=\left(v_{1} \oplus u_{1}, \ldots, v_{q} \oplus u_{q}\right)$.

Заметим, что выполнено неравенство треугольника

$$
|v \oplus u|=\sum_{i=1}^{l} v_{i} \oplus u_{i} \leqslant \sum_{i=1}^{l}\left(v_{i}+u_{i}\right)=\sum_{i=1}^{l} v_{i}+\sum_{i=1}^{l} u_{i}=|v|+|u| .
$$

Величина $|v \oplus u|$ - количество компонент, по которым векторы $u$ и $v$ различаются.

Пусть схема $K$ имеет $n$ входов.

Потенциалом схемы $K$ на входном наборе $x \in\{0,1\}^{n}$ назовем величину $u_{K}(x):=\left|s_{K}(x)\right|$.

Потенциалом схемы $K$ на множестве входных наборов $D \subseteq\{0,1\}^{n}$ назовем $\widehat{U}_{D}(K):=\max _{x \in D} u_{K}(x)$.

Максимальным потенииалом схемы $K$ назовем величину $\widehat{U}(K):=\widehat{U}_{\{0,1\}^{n}}(K)$.

Величину $w_{K}(x, y):=\left|s_{K}(x) \oplus s_{K}(y)\right|$ назовем затратой энергии на переключение входа с $x$ на $y$. 
Максимальной мощностью схемы $K$ на множестве входных наборов $D \subseteq\{0,1\}^{n}$ назовем величину $\widehat{W}_{D}(K):=\max _{x, y \in D} w_{K}(x, y)$. Обозначим $\widehat{W}(K):=W_{\{0,1\}^{n}}(K)$ и будем называть $\widehat{W}_{D}(K)$ максималъной мощностъю схемы $K$.

Замечание 2. Из (1) и свойства $v \oplus u \oplus u \oplus w=v \oplus w$ следует неравенство треугольника для $w_{K}$

$w_{K}(x, y)+w_{K}(y, z)=\left|s_{K}(x) \oplus s_{K}(y)\right|+\left|s_{K}(y) \oplus s_{K}(z)\right| \geqslant\left|s_{K}(x) \oplus s_{K}(z)\right|=w_{K}(x, z)$,

то есть $w_{K}$ является метрикой на множестве $\{0,1\}^{n}$.

Средняя мощность. Пусть задан некоторый случайный процесс $X(t)$ с дискретным временем $t=0,1,2, \ldots$ и значениями в $\{0,1\}^{n}$. Его можно воспринимать также как последовательность случайных векторов $X(0), X(1), X(2), \ldots$, которую будем называть входной последовательностью.

Средней мощностъю схемы $K$ на входной последовательности $X$ в момент времени $t$ назовем величину

$$
W_{X, t}(K):=\mathbf{E} w_{K}(X(t-1), X(t)):=\sum_{x, y \in\{0,1\}^{n}} \mathbf{P}\{X(t-1)=x, X(t)=y\} w_{K}(x, y) .
$$

Средней мощностъю схемы $K$ на входной последовательности $X$ в промежуток времени от $t_{1}$ до $t_{2}$ назовем величину

$$
W_{X, t_{1}, t_{2}}(K):=\frac{1}{t_{2}-t_{1}} \sum_{t=t_{1}+1}^{t_{2}} W_{X, t}(K) \text {. }
$$

Средней мощностъю схемы $K$ на входной последовательности $X$ назовем величину $W_{X}(K):=\varlimsup_{T \rightarrow \infty} W_{X, 0, T}(K)$.

Аналогичные определения можно сформулировать и для потенциала.

Средним потенииалом схемы $K$ на входной последовательности $X$ в момент времени $t$ назовем величину

$$
U_{X, t}(K):=\mathbf{E} u_{K}(X(t)):=\sum_{x \in\{0,1\}^{n}} P\{X(t)=x\} u_{K}(x) .
$$

Средним потенииалом схемы $K$ на входной последовательности $X$ в промежуток времени от $t_{1}$ до $t_{2}$ назовем величину

$$
U_{X, t_{1}, t_{2}}(K)=\frac{1}{t_{2}-t_{1}} \sum_{t=t_{1}+1}^{t_{2}} U_{X, t}(K)
$$

Средним потенииалом схемы $K$ на входной последовательности $X$ назовем величину

$$
U_{X}(K):=\varlimsup_{T \rightarrow \infty} U_{X, 0, T}(K) .
$$

Далее мы будем рассматривать случай, когда векторы $X(0), X(1), \ldots$ независимы и равномерно распределены. В этом случае индекс $X$ в обозначениях мощности и 
потенциала опустим, то есть среднюю мощность будем обозначать $W(K)$, средний потенциал - $U(K)$.

Стандартным способом вводим мощность и потенциал булевых операторов. Если $H(K)$ - некоторая мера мощности, $F:\{0,1\}^{n} \rightarrow\{0,1\}^{m}$ - булев оператор, то

$$
H(F):=\min _{K: F_{K}=F} H(K) .
$$

Введем функции Шеннона для всех мер мощности. Через $P_{2}(n, m)$ обозначим множество всех булевых операторов с $n$ входами и $m$ выходами, через $P_{2}(n):=$ $P_{2}(n, 1)$ - множество булевых функций от $n$ переменных,

$$
\begin{aligned}
\widehat{W}(n, m):=\max _{F \in P_{2}(n, m)} \widehat{W}(F), \quad \widehat{U}(n, m):=\max _{F \in P_{2}(n, m)} \widehat{U}(F), \\
W(n, m):=\max _{F \in P_{2}(n, m)} W(F), \quad U(n, m):=\max _{F \in P_{2}(n, m)} U(F) .
\end{aligned}
$$

И отдельно для булевых функций

$$
\begin{aligned}
\widehat{W}(n):=\max _{f \in P_{2}(n)} \widehat{W}(f), \quad \widehat{U}(n):=\max _{f \in P_{2}(n)} \widehat{U}(f), \\
W(n):=\max _{f \in P_{2}(n)} W(f), \quad U(n):=\max _{f \in P_{2}(n)} U(f) .
\end{aligned}
$$

Теорема 1. Имеют место соотношения

$$
\begin{gathered}
\widehat{U}(n, m)-m \leqslant \widehat{W}(n, m) \leqslant 2 \widehat{U}(n, m), \\
U(n, m)-m-n \leqslant W(n, m) \leqslant 2 U(n, m) .
\end{gathered}
$$

Теорема 2. Имеют место следуюшие утверждения:

1) $\widehat{W}(n) \asymp W(n) \asymp \widehat{U}(n) \asymp U(n) \asymp 2^{n / 2}$;

2) существует такая константа $C>0$, что доля функиий $f$ от $n$ переменных, для которьх $W(f)<C 2^{n / 2}$, стремится $\kappa 0$ при $n \rightarrow \infty$;

3) для любой функиии $f$ от $n$ переменных существует такая реализующая ее схема $K$, что $\widehat{W}(K) \leqslant 9 \sqrt{2} \cdot 2^{n / 2}$ и $S(K) \sim 2^{n}$ nрu $n \rightarrow \infty$.

\section{3. Связь разных мер мощности.}

Лемма 1. Пусть $X(0), X(1), X(2), \ldots$ независимы и одинаково распределень, $P$ вероятностная мера на $\{0,1\}^{n}$, то есть $P(x)=\mathbf{P}\{X(t)=x\}$ не зависит от $t$. Тогда для любой схемы $K$ с $n$ входами

$$
\begin{array}{r}
W_{X}(K)=\sum_{x, y \in\{0,1\}^{n}} P(x) P(y) w_{K}(x, y), \quad W(K)=\frac{1}{2^{2 n}} \sum_{x, y \in\{0,1\}^{n}} w_{K}(x, y), \\
U_{X}(K)=\sum_{x \in\{0,1\}^{n}} P(x) u_{K}(x), \quad U(K)=\frac{1}{2^{n}} \sum_{x \in\{0,1\}^{n}} u_{K}(x) .
\end{array}
$$

Доказательство. Из независимости $X(t-1)$ и $X(t)$ получаем, что

$$
W_{X, t}(K)=\sum_{x, y \in\{0,1\}^{n}} \mathbf{P}\{X(t-1)=x, X(t)=y\} w_{K}(x, y)=\sum_{x, y \in\{0,1\}^{n}} P(x) P(y) w_{K}(x, y) .
$$


Отсюда

$$
W_{X}(K)=\varlimsup_{T \rightarrow \infty} W_{X, 0, T}(K)=\varlimsup_{T \rightarrow \infty} \frac{1}{T} \sum_{t=1}^{T} W_{X, t}(K)=\sum_{x, y \in\{0,1\}^{n}} P(x) P(y) w_{K}(x, y) .
$$

Аналогично для потенциала $U_{K}(t)$ : не зависит от $t$, поэтому

$$
U_{X}(K)=U_{X, t}(K)=\mathbf{E} u_{K}(X(t))=\sum_{x \in\{0,1\}^{n}} P(x) u_{K}(x) .
$$

Здесь даже не требуется независимости входных наборов. Подставляя равномерное распределение, т.е. $P(x)=\frac{1}{2^{n}}$, получаем выражения для $W(K)$ и $U(K)$.

Лемма 2. Для любой входной последовательности $X$ и любой схемы $K$ выполнено

$$
W_{X}(K) \leqslant \widehat{W}(K) \leqslant 2 \widehat{U}(K) .
$$

Доказательство. Так как $w_{K}(x, y) \leqslant \widehat{W}(K)$, то

$$
\mathbf{E} \sum_{t=t_{1}+1}^{t_{2}} w_{k}(X(t-1), X(t)) \leqslant \sum_{t=t_{1}+1}^{t_{2}} \widehat{W}(K)=\left(t_{2}-t_{1}\right) \widehat{W}(K) .
$$

Поэтому $W_{X, t_{1}, t_{2}}(K) \leqslant \widehat{W}(K)$; переходя к пределу, получаем $W(K) \leqslant \widehat{W}(K)$. Далее,

$$
\begin{gathered}
w_{K}(x, y)=\left|s_{K}(x) \oplus s_{K}(y)\right| \leqslant\left|s_{K}(x)\right|+\left|s_{K}(y)\right|=u_{K}(x)+u_{K}(y) \Rightarrow \\
\Rightarrow \widehat{W}_{D}(K)=\max _{x, y \in D} w_{K}(x, y) \leqslant \max _{x, y \in D}\left(u_{K}(x)+u_{K}(y)\right)=2 U_{D}(K) .
\end{gathered}
$$

Отсюда при $D=\{0,1\}^{n}$ получаем $\widehat{W}(K) \leqslant 2 \widehat{U}(K)$.

Существует также связь между средней мощностью и средним потенциалом.

Лемма 3. Для произвольной входной последовательности $X(0), X(1), \ldots$ схемъ $K$ u любого момента времени $t \in \mathbb{N}$ справедливы неравенства

$$
W_{X, t}(K) \leqslant U_{X, t-1}(K)+U_{X, t}(K), \quad W_{X}(K) \leqslant 2 U_{X}(K) .
$$

Доказательство. Пусть

$$
P_{t}(x):=\mathbf{P}\{X(t)=x\}, \quad P_{t}(x, y):=\mathbf{P}\{X(t-1)=x, X(t)=y\} .
$$

Поскольку событие $\{X(t)=y\}$ представляется в виде дизъюнктного объединения событий

$$
\{X(t)=y\}=\bigsqcup_{x \in\{0,1\}^{n}}\{X(t-1)=x, X(t)=y\}, \text { то } \sum_{x \in\{0,1\}^{n}} P_{t}(x, y)=P_{t}(y) .
$$

Аналогично $\sum_{y \in\{0,1\}^{n}} P_{t}(x, y)=P_{t-1}(x)$. Учитывая, что $w_{K}(x, y) \leqslant u_{K}(x)+u_{K}(y)$, получаем

$$
\begin{aligned}
W_{X, t}(K) & =\sum_{x, y \in\{0,1\}^{n}} P_{t}(x, y) w_{K}(x, y) \leqslant \sum_{x, y \in\{0,1\}^{n}} P_{t}(x, y)\left(u_{K}(x)+u_{K}(y)\right)= \\
= & \sum_{x \in\{0,1\}^{n}} \sum_{y \in\{0,1\}^{n}} P_{t}(x, y) u_{K}(x)+\sum_{y \in\{0,1\}^{n}} \sum_{x \in\{0,1\}^{n}} P_{t}(x, y) u_{K}(y)= \\
& =\sum_{x \in\{0,1\}^{n}} P_{t-1}(x) u_{K}(x)+\sum_{y \in\{0,1\}^{n}} P_{t}(y) u_{K}(y)=U_{X, t-1}(K)+U_{X, t}(K) .
\end{aligned}
$$


Для средних значений по отрезку имеем

$W_{X, 0, t}(K)=\frac{1}{t} \sum_{s=1}^{t} W_{X, s}(K) \leqslant \frac{1}{t} \sum_{s=1}^{t}\left(U_{X, s-1}(K)+U_{X, s}(K)\right) \leqslant \frac{1}{t} U_{X, 0}(K)+2 U_{X, 0, t}(K)$.

Переходя к верхнему пределу по $t \rightarrow \infty$, получим

$$
W_{X}(K) \leqslant \lim _{t \rightarrow \infty} \frac{1}{t} U_{X, 0}(K)+2 \varlimsup_{t \rightarrow \infty} U_{X, 0, t}(K)=2 U_{X}(K) .
$$

Существуют также оценки сверху $\widehat{U}$ через $\widehat{W}$ и $U$ через $W$. Для того, чтобы их сформулировать, потребуются определения.

Схема $K_{1}$ подобна схеме $K_{2}$ (обозначение $K_{1} \sim K_{2}$ ), если выполняются следующие условия.

- Графы схем $K_{1}$ и $K_{2}$ с учетом укладки на плоскость совпадают. Тем самым можно ввести общую для $K_{1}$ и $K_{2}$ нумерацию узлов. Числа входов у $K_{1}$ и $K_{2}$ также должны совпадать, обозначим их через $n$.

- Существует такой вектор $C$, что $s_{K_{1}}(x) \oplus s_{K_{2}}(x)=C$ для любого $x \in\{0,1\}^{n}$.

Скажем, что нумерация узлов схемы согласована с порядком вычисления, если для любого ребра номер его начала меньше, чем номер конца. Такая нумерация существует в силу того, что граф схемы по определению не имеет ориентированных циклов.

Лемма 4. Если схемы $K_{1}$ и $K_{2}$ подобны, то их мощность одинакова, то есть

$$
w_{K_{1}} \equiv w_{K_{2}}, W\left(K_{1}\right)=W\left(K_{2}\right),
$$

$u \widehat{W}_{D}\left(K_{1}\right)=\widehat{W}_{D}\left(K_{2}\right)$ для любого множества $D \subseteq\{0,1\}^{n}$.

Доказательство. Пусть $C:=s_{K_{1}}(x) \oplus s_{K_{2}}(x)$. Поскольку $K_{1} \sim K_{2}$, постольку $C$ не зависит от $x$. Поэтому для любых входов $x, y$

$$
\begin{gathered}
0=C \oplus C=s_{K_{1}}(x) \oplus s_{K_{2}}(x) \oplus s_{K_{1}}(y) \oplus s_{K_{2}}(y)=\left(s_{K_{1}}(x) \oplus s_{K_{1}}(y)\right) \oplus\left(s_{K_{2}}(x) \oplus s_{K_{2}}(y)\right) \\
w_{K_{1}}(x, y)=s_{K_{1}}(x) \oplus s_{K_{1}}(y)=s_{K_{2}}(x) \oplus s_{K_{2}}(y)=w_{K_{2}}(x, y) .
\end{gathered}
$$

Так как $W$ и $\widehat{W}_{D}$ являются функционалами от $w$, то $W\left(K_{1}\right)=W\left(K_{2}\right), \widehat{W}_{D}\left(K_{1}\right) \equiv$ $\widehat{W}_{D}\left(K_{2}\right)$.

Лемма 5. Если $l-$ число узлов схемы $K$, то для любого $C \in\{0,1\}^{l}$ существует такая схема $K^{\prime}$, что $K^{\prime} \sim K u s_{K_{1}}(x) \oplus s_{K_{2}}(x \oplus \vec{c})=C$, где $\vec{c}-$ вектор, составленный из компонент $C$, соответствующих входам схемы $K$.

Доказательство. Если схема $K^{\prime}$ удовлетворяет условиям леммы, то будем писать $K^{\prime}=K \oplus C$.

Пусть имеется схема $K$. Выберем нумерацию узлов, согласованную с порядком вычисления. Зафиксируем некоторое $C \in\{0,1\}^{l}$. Покажем, что можно заменить функции элементов схемы $K$ так, чтобы для полученной схемы $K^{\prime}$ было выполнено $s_{K^{\prime}} \oplus s_{K}=C$ или, что то же самое, $s_{K^{\prime}}=s_{K} \oplus C$. Обозначим $\left(g_{1}, \ldots, g_{l}\right)$ значения в узлах схемы $K$. 
Рассмотрим произвольный элемент $e$ схемы $K$. Пусть $g_{i_{1}}, \ldots, g_{i_{m}}$ - входы элемента $e$, а $g_{o_{1}}, \ldots, g_{o_{q}}-$ выходы $(m+q \leqslant 4)$. Элемент $e$ по определению представляет множество функций $f_{1}, \ldots, f_{k}, k \leqslant q$, зависящих от $g_{i_{1}}, \ldots, g_{i_{m}}$, причем каждому выходу $g_{o_{j}}$ соответствует некоторая функция $f_{l_{j}}$. В схеме $K^{\prime}$ на месте элемента $e$ будет стоять элемент $e^{\prime}$ с таким же расположением входов и выходов, но $j$-му выходу будет соответствовать функция

$$
f_{l_{j}}^{\prime}\left(g_{i_{1}}, \ldots, g_{i_{m}}\right)=f_{l_{j}}\left(g_{i_{1}} \oplus C_{i_{1}}, \ldots, g_{i_{m}} \oplus C_{i_{m}}\right) \oplus C_{o_{j}} .
$$

Тогда для любого элемента $e$ номер каждого выхода больше номеров всех входов, от которого этот выход зависит. Теперь можно проверить по индукции, что $g_{i}^{\prime}(x \oplus \vec{c})=g_{i}(x) \oplus C_{i}$.

Пусть для всех $0<j<i$ выполнено $g_{j}^{\prime}(x \oplus \vec{c})=g_{j}(x) \oplus C_{j}$. Рассмотрим два случая:

1) $g_{i}$ является $l$-м входом схемы, тогда $g_{i}^{\prime}(x \oplus \vec{c})=x_{l} \oplus c_{l}=g_{i}(x) \oplus C_{i}$;

2) $g_{i}$ является выходом некоторого элемента $e$. Тогда ему соответствует некоторая функция $f\left(g_{i_{1}}, \ldots, g_{i_{n}}\right)$, где $g_{i_{1}}, \ldots, g_{i_{n}}$ - входы элемента $e$, причем $i_{1}, \ldots, i_{n}<i$, так как нумерация согласована с порядком вычисления. Учитывая предположение индукции, получаем

$$
\begin{gathered}
g_{i}^{\prime}(x \oplus \vec{c})=f^{\prime}\left(g_{i_{1}}^{\prime}(x \oplus \vec{c}), \ldots, g_{i_{n}}^{\prime}(x \oplus \vec{c})\right)=f^{\prime}\left(g_{i_{1}}(x) \oplus C_{i_{1}}, \ldots, g_{i_{n}}(x) \oplus C_{i_{n}}\right)= \\
f\left(g_{i_{1}}(x) \oplus C_{i_{1}} \oplus C_{i_{1}}, \ldots, g_{i_{n}}(x) \oplus C_{i_{n}} \oplus C_{i_{n}}\right) \oplus C_{i}=f\left(g_{i_{1}}(x), \ldots, g_{i_{n}}(x)\right) \oplus C_{i}=g_{i}(x) \oplus C_{i} .
\end{gathered}
$$

Лемма 6. Для любой схемы $K$ с $n$ входами и $m$ выходами и набора $x_{0} \in\{0,1\}^{n}$ существует такая подобная ей схема $K_{x_{0}}$, что $F_{K}=F_{K_{x_{0}}}$ u для всех $x \in\{0,1\}^{n}$

$$
u_{K_{x_{0}}}(x) \leqslant w_{K_{x_{0}}}\left(x, x_{0}\right)+m+\left|x_{0}\right| .
$$

Доказательство. Для построения схемы $K^{\prime}$ будем использовать предыдущую лемму. Положим $K+x_{0}=K \oplus C$, где

$$
C_{i}= \begin{cases}0, & i \text {-й узел является входом или выходом } K ; \\ \left(s_{K}\left(x_{0}\right)\right)_{i}, & \text { в остальных случаях. }\end{cases}
$$

Тогда на входах и выходах у схем $K$ и $K_{x_{0}}$ будут реализовываться одинаковые функции, то есть $\vec{c}=0$ и $F_{K}=F_{K_{x_{0}}}$. Далее

$\left(s_{K_{x_{0}}}\left(x_{0}\right)\right)_{i}= \begin{cases}\left(s_{K}\left(x_{0}\right)\right)_{i}, & i \text {-й узел является входом или выходом } K ; \\ \left(s_{K}\left(x_{0}\right)\right)_{i} \oplus\left(s_{K}\left(x_{0}\right)\right)_{i}=0 & \text { в остальных случаях. }\end{cases}$

Поэтому в векторе $s_{K_{x_{0}}}\left(x_{0}\right)$ не более $m+\left|x_{0}\right|$ ненулевых компонент, то есть $\left|s_{K_{x_{0}}}\left(x_{0}\right)\right| \leqslant m+\left|x_{0}\right|$. Из неравенства треугольника для $s_{K_{x_{0}}}(x)=\left(s_{K_{x_{0}}}(x) \oplus\right.$ $\left.s_{K_{x_{0}}}\left(x_{0}\right)\right) \oplus s_{K_{x_{0}}}\left(x_{0}\right)$ получаем

$$
u_{K_{x_{0}}}(x)=\left|s_{K_{x_{0}}}(x)\right| \leqslant\left|s_{K^{\prime}}(x) \oplus s_{K_{x_{0}}}\left(x_{0}\right)\right|+\left|s_{K_{x_{0}}}\left(x_{0}\right)\right| \leqslant w_{K_{x_{0}}}\left(x, x_{0}\right)+m+\left|x_{0}\right| .
$$

Лемма 7. Для любой схемы $K$ с $n$ входами и $m$ выходами существует такая подобная ей схема $K^{\prime}$, что $F_{K}=F_{K^{\prime}}$ и для любого множества $D \subseteq\{0,1\}^{n}$

$$
\widehat{U}_{D}\left(K^{\prime}\right) \leqslant \widehat{W}_{D}(K)+m+\min _{x \in D}|x| .
$$


Доказательство. Положим $x_{0}=\arg \min _{x \in D}|x|$, то есть $x_{0}$ - вектор из $D$ с наименьшим числом единиц. Положим $K^{\prime}=K_{x_{0}}$. Тогда $F_{K^{\prime}}=F_{K}$ и для всех $x \in D$ выполнено $u_{K^{\prime}}(x) \leqslant w_{K}\left(x, x_{0}\right)+m+\left|x_{0}\right|$ по лемме 6. Отсюда

$$
\widehat{U}_{D}\left(K^{\prime}\right)=\max _{x \in D} u_{K^{\prime}}(x) \leqslant \max _{x \in D} w_{K^{\prime}}\left(x, x_{0}\right)+m+\left|x_{0}\right| \leqslant \widehat{W}_{D}\left(K^{\prime}\right)+m+\left|x_{0}\right| .
$$

Учитывая, что $\left|x_{0}\right|=\min _{x \in D}|x|$, и $\widehat{W}_{D}\left(K^{\prime}\right)=\widehat{W}_{D}(K)$ по лемме 4 , получим требуемое неравенство.

Лемма 8. Если входные наборы $X(0), X(1), X(2), \ldots$ независимъ и одинаково распределены, то существует такая схема $K^{\prime}$, что $K^{\prime} \sim K, F_{K}=F_{K^{\prime}} u$

$$
U_{X}\left(K^{\prime}\right) \leqslant W_{X}(K)+m+n .
$$

Доказательство. Пусть $P(x)$ - вероятность входного набора $x$. Поскольку входы одинаково распределены, $P(x)$ не зависит от времени. Пусть

$$
x_{0}:=\arg \min _{y \in\{0,1\}^{n}} \sum_{x \in\{0,1\}^{n}} P(x) w_{K}(x, y) .
$$

Положим $K^{\prime}=K_{x_{0}}$, тогда $F_{K^{\prime}}=F_{K}$ и для всех $x \in D$ выполнено $u_{K^{\prime}}(x) \leqslant w_{K}\left(x, x_{0}\right)+m+\left|x_{0}\right|$ по лемме 6 . Учитывая, что $\left|x_{0}\right| \leqslant n$, получаем

$$
\begin{aligned}
& U\left(K^{\prime}\right)-m-n=\sum_{x \in\{0,1\}^{n}} P(x)\left(u_{K^{\prime}}(x)-m-n\right) \leqslant \sum_{x \in\{0,1\}^{n}} P(x) w_{K}\left(x, x_{0}\right)= \\
& =\min _{y \in\{0,1\}^{n}} \sum_{x \in\{0,1\}^{n}} P(x) w_{K}(x, y) \leqslant \sum_{y \in\{0,1\}^{n}} P(y) \sum_{x \in\{0,1\}^{n}} P(x) w_{K}(x, y)=W(K) .
\end{aligned}
$$

Доказательство теоремы 1. Из лемм $1-8$ вытекает, что для всех $F \in P_{2}(n, m)$

$$
\begin{gathered}
\widehat{U}(F)-m \leqslant \widehat{W}(F) \leqslant 2 \widehat{U}(F), \\
U(F)-m-n \leqslant W(F) \leqslant 2 U(F) .
\end{gathered}
$$

Отсюда, переходя к максимуму по $F$, сразу получаем утверждение теоремы 1.

Таким образом, максимальный потенциал и максимальная мощность - величины одного порядка, если нет ограничений на базис. А если входы схемы независимы и одинаково распределены, то средний потенциал и средняя мощность - величины одного порядка.

\section{4. Верхняя оценка мощности}

Единичным подграфом схемы $K$ на входном наборе $x$ назовем подграф схемы $K$, состоящий из всех узлов, значение в которых равно 1, и всех ребер, их соединяющих.

Скажем, что клеточный элемент сохраняет 0, если при подаче на все его входы значения 0 на всех его выходах выдаются значения 0.

Для доказательства верхней оценки будем строить схему с требуемым потенциалом, потом воспользуемся оценкой (5) мощности через потенциал. При подсчете потенциала будем использовать следующую лемму. 
Лемма 9. Если все клеточные элементы схемы $K$ сохраняют 0, то для любого входного набора $x$ каждая компонента связности единичного подграфа содержит хотя бы один вход схемы $K$.

Доказательство. Зафиксируем произвольный входной набор $x$. Допустим, что есть компонента связности $G_{0}$ единичного подграфа, в которой нет ни одного входа схемы $K$. Поскольку весь граф схемы не содержит ориентированных циклов, то $G_{0}$ также их не содержит. Значит, есть вершина $v$ графа $G_{0}$, в которую не входит ни одного ребра. Поскольку $v$ по предположению не является входом схемы $K$, она является выходом некоторого элемента $e$. Пусть выход $v$ зависит от входов $v_{1}, \ldots, v_{q}$ элемента $e$, т.е. $g_{v}=f\left(g_{v_{1}}, \ldots, g_{v_{q}}\right)$ (где $g_{u}-$ значение в узле $u$ ). Тогда в графе схемы присутствуют ребра $v_{1} v, \ldots, v_{q} v$, но они не являются ребрами $G_{0}$, так как в нём нет ребер, входящих в вершину $v$. Значит, по определению единичного подграфа, узлы $v_{1}, \ldots, v_{q}$ не являются вершинами $G_{0}$, то есть значения $g_{v_{1}}, \ldots, g_{v_{q}}$ равны 0 . Но поскольку $f$ сохраняет 0 , то $g_{v}=f\left(g_{v_{1}}, \ldots, g_{v_{q}}\right)=f(0, \ldots, 0)=0$ - противоречие с тем, что $v$ - вершина единичного подграфа.

Эта лемма позволяет при подсчете потенциала рассматривать узлы схемы, начиная от единичных входов, и смотреть, куда проходит единичный сигнал от этих входов.

При построении будем использовать операцию удаления ненужных выходов.

Лемма 10. Для любой плоской схемы $K$ и любого подмножества ее выходов $U$ существует схема $K_{U}$, носитель которой лежит в носителе схемы $K$, множество ее выходов совпадает с $U$ и на каждом из этих выходов $K_{U}$ реализует ту же функиию, что и схема $K$.

Доказательство. Следующий алгоритм позволяет построить схему $K_{U}$, указанную в лемме.

\section{Алгоритм удаления ненужных выходов.}

Пусть $V=O u t(K) \backslash U-$ множество выходов схемы $K$, не лежащих в $U$. Сначала положим $K_{U}=K$. Пока множество $V$ не пусто, выполняем следующее.

(1) Выберем произвольно $v \in V$.

(2) Исключим $v$ из множества $V$.

(3) Если $v$ совпадает со входом схемы $K_{U}$, то удалим узел $v$ из схемы $K_{U}$ и перейдем к началу цикла;

иначе $v$ - выход некоторого элемента $e$.

(4) Если $v$ - единственный выход элемента $e$, то

(a) добавим в множество $V$ все входы элемента $e$;

(b) удалим элемент $e$ из схемы $K_{U}$.

(5) Иначе удалим из элемента $e$ выход $v$, то есть соответствующая сторона $e$ станет изолятором. 
Алгоритм обязательно завершит работу, поскольку на каждом шаге мы либо удаляем один узел схемы $K$ (если $v$ не является входом схемы), либо количество элементов в $V$ уменьшаем на 1 (если $v$ совпало со входом схемы). Поэтому количество шагов не превышает количество узлов схемы $K$.

Заметим, что перед каждым шагом алгоритма $V \cup U=\operatorname{Out}\left(K_{U}\right)$ :

- Перед первым шагом это выполнено по определению множества $V$.

- На каждом шаге узел $v$ исключается из множества $V$ и одновременно удаляется из схемы $K$.

- Если $v$ - единственный выход элемента $e$, то элемент $e$ удаляется из схемы, и все его входы становятся выходами схемы. Вместе с этим они добавляются к множеству $V$.

- Если $v$ - не единственный выход элемента $e$, из схемы $K$ удаляется лишь узел $v$, и новых выходов не образуется.

Таким образом, на каждом шаге алгоритма соотношение $V \cup U=O u t(K)$ сохраняется. Таким образом, после завершения работы $V$ пусто, значит, $U=\operatorname{Out}\left(K_{U}\right)$.

При работе этого алгоритма лишь удаляются узлы схемы, но не изменяются функции, реализуемые элементами в оставшихся узлах. Поэтому для любого входного набора состояние схемы $K_{U}$ является проекцией состояния схемы $K$ на множество узлов $K_{U}$. Поэтому потенциал и мощность схемы $K_{U}$ не превосходят соответственно потенциала и мощности схемы $K$.

Эта лемма позволяет при построении схемы использовать одинаковые элементы или блоки и не рассматривать отдельно крайние элементы или блоки схемы, выходы которых могут стать лишними выходами схемы.

Лемма 11. Если $f$ - булева функция от $n$ переменных, то существует такая схема $K$, реализующая функиию $f$, что

$$
\begin{gathered}
\widehat{W}(K) \lesssim 9 \sqrt{2} \cdot 2^{n / 2} \text { nрu } n \rightarrow \infty, \\
S(K) \sim 2^{n} \text { nри } n \rightarrow \infty .
\end{gathered}
$$

Доказательство. При методе, описанном в [2], которым строится схема площадью $2^{n}$, мощность получается порядка $n \cdot 2^{n / 2}$, причем она в основном выделяется на дешифраторе (блоке, реализующем все элементарные конъюнкции). При построении схемы можно использовать те же блоки, что и для построения схемы в [2, лемма 3], добавив блок, снижающий мощность дешифратора. Но мы немного изменим все блоки, немного уменьшая площадь, используя элементы, реализующие одновременно две булевы функции.

Напомним, что прямоугольная схема называется повторяющей, если напротив каждого ее входа $x$ на противоположной стороне симметрично расположен выход $y$, на котором реализуется тождественная функция $y(x)=x$.

Будем описывать элементы схемы следующим образом: чтобы описать элемент $e$ с координатами $(x, y)$, будем писать

$$
(x, y): s_{1}=f_{1}\left(s_{m+1}, \ldots, s_{q}\right), \ldots, s_{m}=f_{m}\left(s_{m+1}, \ldots, s_{q}\right),
$$


где $s_{j} \in\{l, t, r, b\}$, причем out $(e)=\left\{s_{1}, \ldots, s_{m}\right\}$, in $(e)=\left\{s_{m+1}, \ldots, s_{q}\right\}$. Ось y у нас направлена вниз, таким образом, контакт $t$ элемента с координатами $(x, y)$ соединен с контактом $b$ элемента с координатами $(x, y-1)$.

Под $\overline{a_{1} \ldots a_{k}}$ будем понимать число $a=\sum_{j=1}^{k} a_{j} 2^{k-j}$.

Введем операцию транспонирования схемы. Транспонированный клеточный элемент $e$ - элемент $\operatorname{Transp}(e)$, получающийся из $e$, если поменять местами метки $l \leftrightarrow t$ и $r \leftrightarrow b$. Транспонированная схема $K-$ схема $K^{T}=\operatorname{Transp\circ } K \circ T$, где $T: \mathbb{Z}^{2} \rightarrow \mathbb{Z}^{2}$, $T(x, y)=(y, x)$. По сути, это схема, получающаяся отражением схемы $K$ относительно прямой $y=x$.

Опишем блоки, которые будем использовать.

(1) $K I\left(a_{1}, \ldots, a_{k}\right)$ - повторяющая схема размером $1 \times k$, принимающая слева переменные $x_{1}, \ldots, x_{k}$ и выдающая снизу элементарную конъюнкцию $x_{1}^{a_{1}} x_{2}^{a_{2}} \ldots x_{k}^{a_{k}}$ (здесь $x^{a}:=x \oplus a=\left\{\begin{array}{ll}x, & a=0 ; \\ \bar{x}, & a=1 .\end{array}\right.$ ).

$(1,1): r=l, b=\left(l \oplus a_{1}\right)$;

$(1, j): r=l, b=t\left(l \oplus a_{j}\right)$ для $j=2, \ldots, k$.

Площадь этого блока равна $k$.

(2) $Q\left(x_{1}, \ldots, x_{k}\right)$ и $Q_{2}\left(x_{1}, \ldots, x_{k}\right)$ - дешифраторы от $k$ переменных.

$Q\left(x_{1}, \ldots, x_{k}\right)$ строится последовательным соединением блоков $K I\left(a_{1}, \ldots, a_{k}\right)$ для всех $\left(a_{1}, \ldots, a_{k}\right) \in\{0,1\}^{k}$. Его площадь равна $k 2^{k}$. Потенциал не превосходит количества узлов, то есть

$$
\widehat{U}\left(Q\left(x_{1}, \ldots, x_{k}\right)\right) \leqslant k\left(2^{k}+1\right)+(k+1) 2^{k} \leqslant(2 k+1)\left(2^{k}+1\right) .
$$

Если заменить все элементы этой схемы на блоки $2 \times 2$ в базисе $\{\&, \vee, \neg\}$ и удалить правые выходы, то получится схема, изображенная на рис. 2 , которая в 4 раза больше по площади, чем сама схема $Q\left(x_{1}, \ldots, x_{k}\right)$.

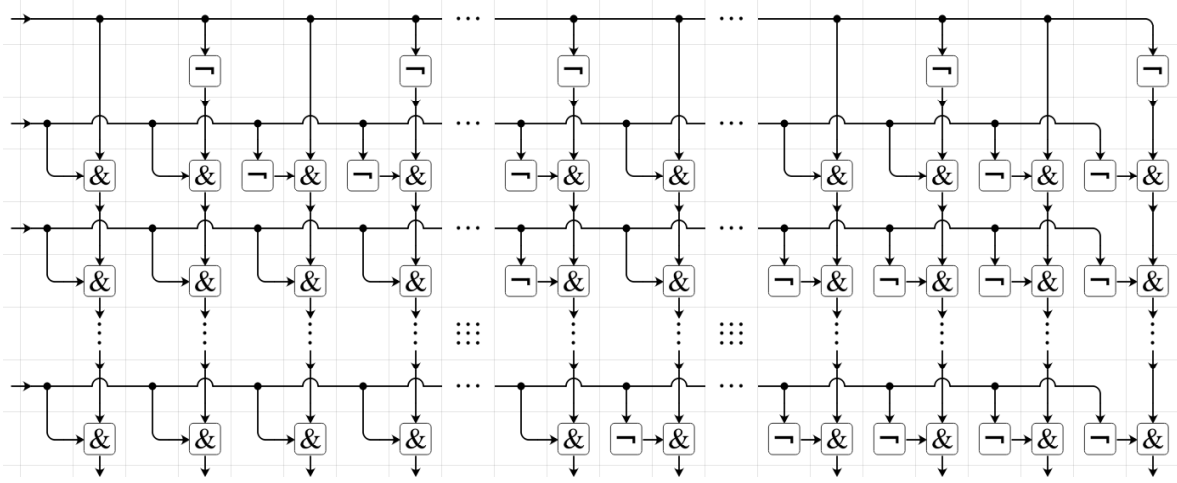

Рис. 2. Схема, реализующая все элементарные конъюнкции от $k$ переменных.

$Q_{2}\left(x_{1}, \ldots, x_{k}\right)$ состоит из блоков $K I\left(a_{1}, \ldots, a_{k}\right)$, стоящих в четных позициях для всех $\left(a_{1}, \ldots, a_{k}\right) \in\{0,1\}^{k}$. На нечетных позициях стоят коммутационные элементы $(r=l)$, которые соединяют $i$-й выход предыдущего блока $K I$ с $i$-м входом 
следующего блока. Площадь такого блока равна $2 k 2^{k}=k 2^{k+1}$. Схема функционирует так же, как и $Q\left(x_{1}, \ldots, x_{k}\right)$, но выходы расположены через 1 , так же, как на рис. 2. Потенциал этой схемы опять же не превосходит количества ее узлов, то есть

$$
\widehat{U}\left(Q_{2}\left(x_{1}, \ldots, x_{k}\right)\right) \leqslant k\left(2^{k+1}+1\right)+(k+1) 2^{k+1} \leqslant(2 k+1)\left(2^{k+1}+1\right) .
$$

(3) $K_{m 1}\left(y_{1}, \ldots, y_{m}, z\right)$ - схема, которая принимает слева входы $y_{1}, \ldots, y_{m}$ и сверху вход $z$. Снизу реализует все конъюнкции вида $y_{i} z$ и справа $y_{1} \bar{z}, \ldots, y_{m} \bar{z}$. Она имеет вид, изображённый на рис. 3 и состоит из элементов типа, изображённого на рис. 4.

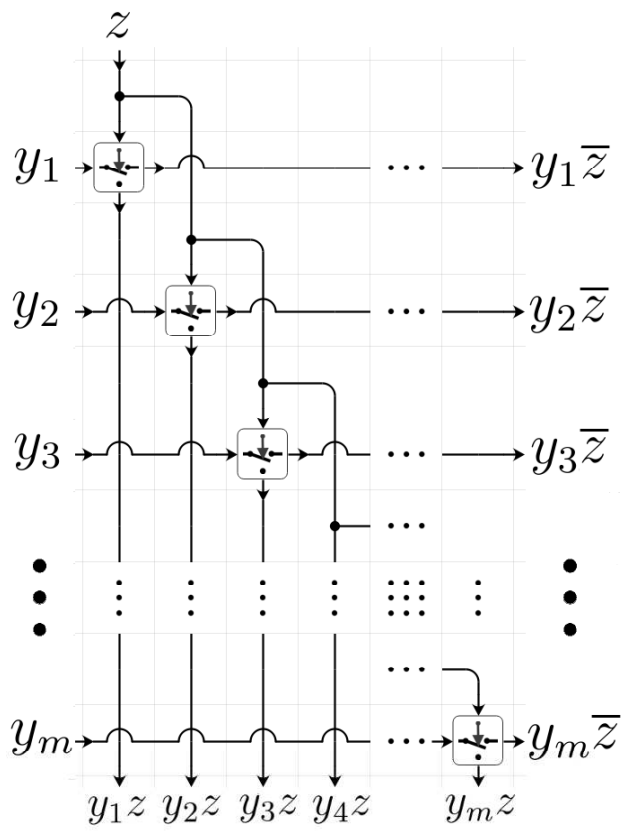

Здесь мы использовали следующий клеточный элемент:

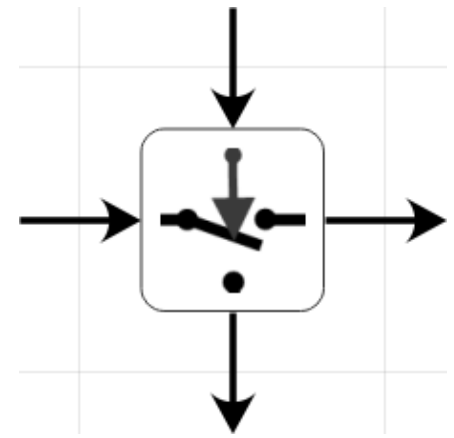

Рис.4: $r=l \& \bar{t}, b=l \& t$

(реле)

Рис.3: Схема $K_{m 1}\left(y_{1}, \ldots, y_{m}, z\right)$.

Пронумеруем все реле: в $2 j$-й позиции по вертикали стоит $j$-е реле (его левый вход соединен с $\left.y_{j}\right)$. Рассмотрим "работу" этого блока:

(a) $j$-й нижний выход соединен через коммутационные элементы с нижним выходом $j$-го реле, на которое подается (через коммутационные элементы) слева вход $y_{j}$, сверху - вход $z$, поэтому на нем реализуется функция $y_{j} z$.

(b) $j$-й правый выход соединен с правым выходом $j$-го реле, на которое подается слева вход $y_{j}$, сверху - вход $z$, поэтому на нем реализуется функция $y_{j} \bar{z}$.

Посчитаем потенциал при условии, что $y_{i}=1, y_{j}=0$ при $j \neq i$. Поскольку коммутационные элементы и реле сохраняют 0, то по лемме 9 можно лишь проследить путь от единичных входов.

(a) $z=0$. Тогда единственный единичный вход $-y_{i}$. Он соединен с левым входом $i$-го реле через коммутационные элементы ( $i$ узлов). Верхний вход $(t)$ 
этого реле соединен с входом $z$, который равен 0 , то есть $l=1, t=0$. Поэтому $b=0, r=1$, то есть на нижнем выходе реле будет 0 , на правом 1 . Правый выход реле соединен только с правым выходом схемы через коммутационные элементы (всего $m-i+1$ узлов). Поэтому единица будет в $m+1$ узлах, то есть потенциал равен $m+1$.

(b) $z=1$. Вход $z$ соединен через коммутационные элементы с $4 m-2$ узлами и подсоединен к верхним входам всех реле (на них подается 1). Поэтому на правом выходе каждого реле выдается 0. Вход $y_{j}$ подсоединен к $j$-му реле, поэтому на нижнем выходе $j$-го реле выдается $y_{j} z=y_{j}=$ $\left\{\begin{array}{ll}1, & j=i, \\ 0, & \text { иначе. }\end{array}\right.$ Вход $y_{i}$ соединен коммутационными элементами с $i$ узлами. Единственный единичный выход реле - нижний выход $i$-го реле, он соединен коммутационными элементами с $(2(m-i)+1)$-м узлом, расположенным непосредственно под $i$-м реле. Таким образом, потенциал равен $4 m-2+i+2(m-i)+1=6 m-i-1<6 m$.

Схема имеет длину $m$ и высоту $2 m$, поэтому ее площадь равна $2 m^{2}$.

(4) Схема $K_{m q}\left(y_{1}, \ldots, y_{m} ; z_{1}, \ldots, z_{q}\right)$ - схема, составленная из $q$ блоков $K_{m 1}$, соединенных последовательно, к верхнему входу $i$-го блока подведена переменная $z_{i}$. На рис. 5 изображена такая схема с удаленными правыми выходами (по лемме 10 удаление выходов не увеличивает мощность и потенциал).

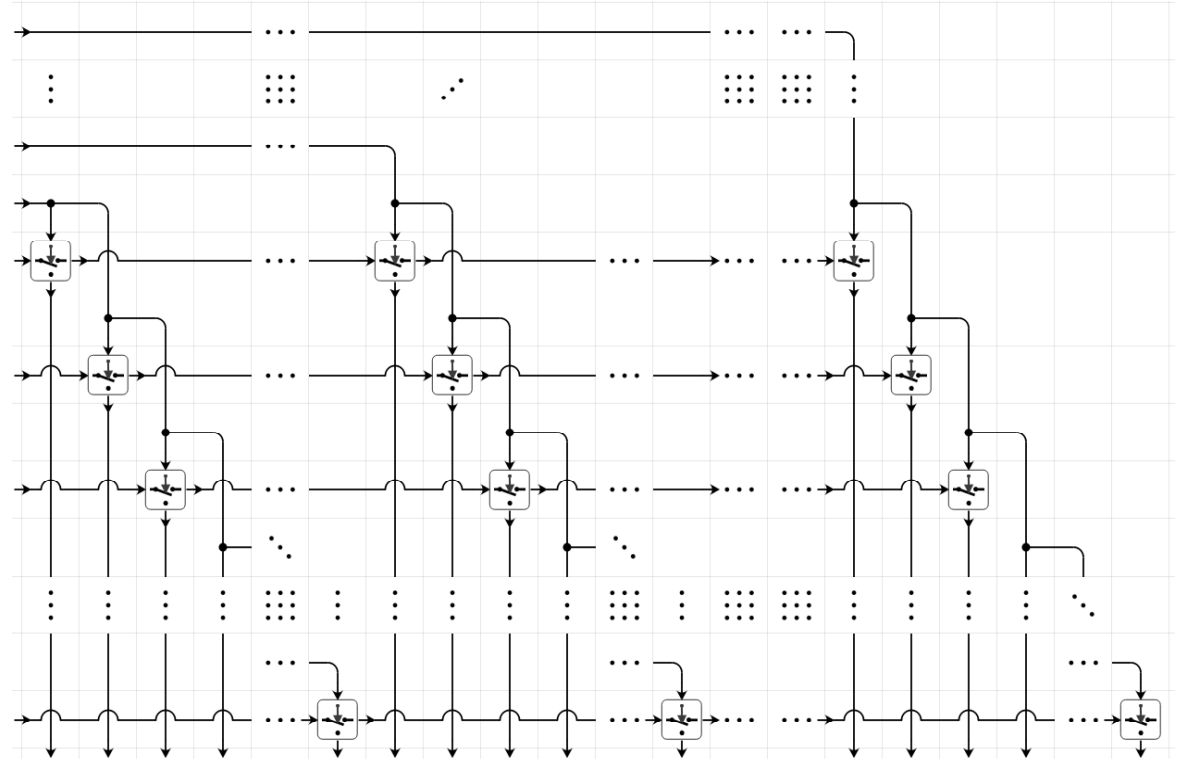

Рис.5: схема $K_{m q}$

Функционирование и мощность схемы будем рассматривать при условии, что ровно одно из $y_{i}$ и ровно одно из $z_{j}$ равно 1 . Покажем, что данная схема выдает на $(j m+i)$-м выходе 1 , а на всех остальных - 0 . 
- Если $y_{i}=1$ и $z_{j}=1$, то на $k$-м блоке $K_{m 1}$ при $k \neq j$ вход $z$ равен 0 , поэтому на нижних выходах этого блока будут нули, а справа тождественные функции, то есть значения на выходах справа будут теми же, что и на входах слева. Потенциал такого блока не более $m+1$, а его вклад в потенциал всей схемы равен $m$, поскольку $i$-й правый выход будет учтен как вход следующего блока.

- На входах $j$-го блока: $z=z_{j}=1$. Входы $y$ будут такими же, как входы схемы, поскольку предыдущие блоки повторяют входные переменные. Значит, $j$-й блок выдаст на $i$-м нижнем выходе 1 , а на остальных - 0 . $i$-й выход $j$-го блока - как раз $(j m+i)$-й выход всей схемы. Потенциал такого блока не превосходит $6 m$.

Осталось подсчитать потенциал провода, подводящего переменную $z_{j}$ к входу $j$-го блока. Длина этого провода по горизонтали равна $(j-1) m+1$, длина по вертикали равна $j$, поэтому такой провод проходит через $(j-1) m+1+j$ узлов. Итак, потенциал всей схемы не превосходит $(q-1) m+6 m+(q-1) m+1+q=$ $(2 q+4) m+1+q=2 q m+4 m+q$. Длина этой схемы равна $m q$, высота равна $2 m+q$, поэтому площадь равна $m q(2 m+q)$. Итак, $i$-й выход $j$-го блока $((j m+i)$-й выход схемы), реализует конъюнкцию $y_{i} z_{j}$.

Мы описали вспомогательные блоки, из которых будем строить дешифратор от $k$ переменных с мощностью порядка $2^{k}$ и площадью порядка $2^{3 k / 2}$ в соответствии с формулой

$$
D_{a}^{k}\left(x_{1}, \ldots, x_{k}\right)=K_{2^{a}, 2^{k}-a}\left(Q\left(x_{1}, \ldots, x_{a}\right), Q\left(x_{a+1}, \ldots, x_{k}\right)\right), \text { где } 1 \leqslant a \leqslant k-1 .
$$

Это возможно при $k \geqslant 2$. Соединять все блоки будем уже на окончательной схеме, изображенной на рис. 6 .

Зафиксируем некоторые натуральные числа $1 \leqslant a<b<c<n$. Обозначим $q=n-b$. Всю схему будем строить на основе СДНФ функции $f$. Для этого сначала построим из описанных блоков схему $K_{l}=D_{a}^{b}\left(x_{1}, \ldots, x_{b}\right)$, которая реализует все элементарные конъюнкции от первых $b$ переменных, и $K_{h}=D_{c-b}^{q}\left(x_{b+1}, \ldots, x_{n}\right)$, реализующую все элементарные конъюнкции от оставшихся $q$ переменных.

Осталась часть схемы $K_{f}$, вычисляющая саму функцию. Эта часть принимает на вход $y_{1}, \ldots, y_{2^{b}}$ (элементарные конъюнкции первой половины входов) и $z_{1}, \ldots, z_{2^{q}}$ (элементарные конъюнкции оставшихся входов), поэтому ровно одно из $y_{i}$ и ровно одно из $z_{j}$ равно 1 (пусть это $y_{i_{0}}$ и $y_{j_{0}}$ ). На выход она выдает $\bigvee_{(i, j) \in L} y_{i} \& z_{j}, L-$ множество индексов, соответствующее единицам функции $f$. Для удобства обозначим $l$-й знак от конца в двоичной записи числа $i$ через $i_{l}$. Тогда $y_{i}=\bigwedge_{l=1}^{b} x_{l}^{i_{l}}, z_{i}=\bigwedge_{l=1}^{q} x_{b+l}^{i_{l}}$, $L=\left\{(i, j): f\left(i_{1}, \ldots, i_{b}, j_{1}, \ldots, j_{q}\right)=1\right\}$, поэтому

$$
\bigvee_{(i, j) \in L} y_{i} \& z_{j}=\bigvee_{(i, j) \in L} \bigwedge_{l=1}^{b} x_{l}^{i_{l}} \bigwedge_{l=1}^{q} x_{b+l}^{j_{l}}=\bigvee_{\sigma: f\left(\sigma_{1}, \ldots, \sigma_{n}\right)=1} \bigwedge_{l=1}^{n} x_{l}^{i_{l}}=f\left(x_{1}, \ldots, x_{n}\right) .
$$

Таким образом, функция $f$ реализуется формулой

$$
K_{f}\left(D_{a}\left(x_{1}, \ldots, x_{b}\right), D_{c-b}\left(x_{b+1}, \ldots, x_{n}\right)\right) .
$$

Саму схему $K_{f}$ легко описать формально:

$(i, j): r=l, b=\bar{l} \& t,((i, j) \in L)$;

3 Дискретная математика, т.26 №1 
$(i, j): r=l \& \bar{t}, b=\bar{l} \& t$ (блокировка), $((i, j) \notin L) ;$

$\left(2^{q}+1,1\right): b=l$

$\left(2^{q}+1, i\right): b=l \& t,\left(2 \leqslant i \leqslant 2^{m}\right)$.

Для реализации этого блока нам понадобятся логические элементы двух типов, изображённые на рис. 6 .

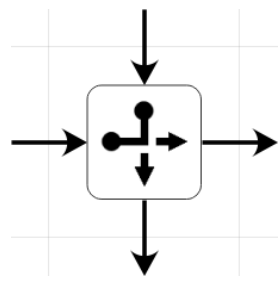

(a) $r=l \& \bar{t}, b=\bar{l} \& t$

(блокировка)

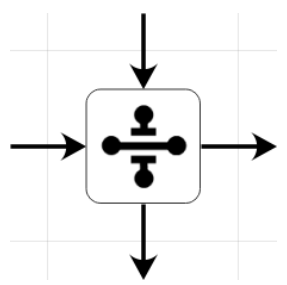

(b) $r=l, b=\bar{l} \& t$

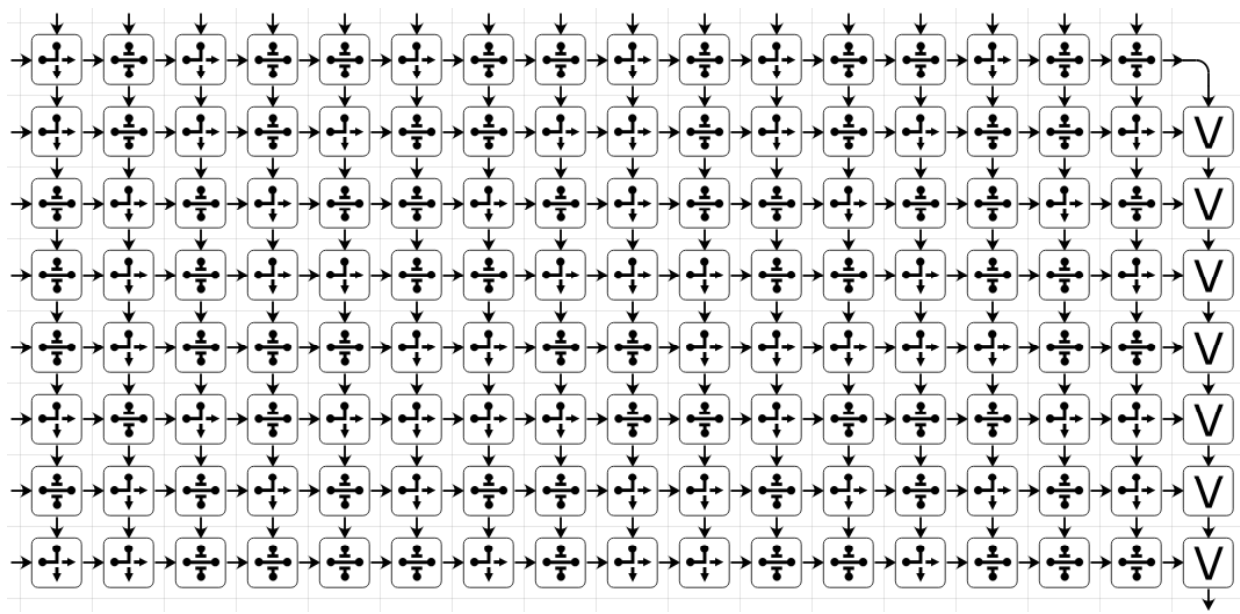

Рис. 6. Пример схемы $K_{f}$ для $b=3, q=4$

Сразу заметим, что все элементы схемы $K_{f}$ сохраняют 0 , поэтому если все входы некоторой ее подсхемы равны 0, то выходы всех элементов этой подсхемы также равны 0 .

Пусть $y_{i}=1, z_{j}=1$. Рассмотрим работу схемы $K_{f}$. Заметим, что оба используемые элементы выдают справа 0 , если на левом входе 0 , и выдают снизу 0 , если на верхнем входе 0 . Поэтому на верхнем входе всех элементов с координатами $(k, l)$ будет 0 при $k \neq j, k \leqslant 2^{q}$, и на левом входе будет 0 , если $l \neq i$. Теперь заметим, что при $t=0$ у обоих элементов $r=l$, а при $l=0$ у обоих элементов $b=t$. Поскольку у всех элементов с координатами $(k, i)$ при $k<j$ на верхний вход подается 0, постольку $r=l=y_{i}=1$. Аналогично у всех элементов с координатами $(j, l)$ при $l<i$ выполняются равенства $b=t=z_{j}=1$. Таким образом, у элемента с координатами $(j, i)$ на оба входа подается 1, и возможны 2 случая.

- $(i, j) \in L$, тогда $r=l=1$ и $b=0$. Тогда у всех элементов $(k, i)$ при $j<k \leqslant 2^{q}$ сверху подается 0 , поэтому будет $r=l=1$, поскольку сверху подается 0 . На все 
входы подсхемы, находящейся в области $\left\{(k, l):\left(1 \leqslant k \leqslant 2^{q}\right) \wedge\left(j<l \leqslant 2^{b}\right)\right\}$, подается 0, поэтому все ее элементы будут выдавать 0 , так как они все сохраняют 0 .

Остается рассмотреть элементы с координатами $\left(2^{q}+1, l\right)$. На все входы блока $\left\{\left(2^{q}+1, l\right), l<j\right\}$ подается 0, поэтому на выходах всех его элементов будет 0 . Левый вход элемента $\left(2^{q}+1, i\right)$ равен 1 , поэтому он на выходе выдаст 1, и вся цепочка дизъюнкций $\left\{\left(2^{q}+1, l\right), l>j\right\}$ выдаст 1 на выходе схемы.

В этом случае единицы на левых входах элементов с координатами $(k, i)$ при $1 \leqslant k \leqslant 2^{q}+1$ (всего $\left(2^{q}+1\right)$ узлов), на верхних контактах элементов $(j, l)$ при $l \leqslant i$ (всего $i$ узлов) и на нижних контактах элементов $\left(2^{q}+1, l\right)$ при $1 \leqslant l \geqslant i$ (всего $2^{b}-i+1$ узлов). Таким образом, потенциал схемы равен $\left(2^{q}+1\right)+i+\left(2^{b}-i+1\right)=2^{q}+2^{b}+2$.

- $(i, j) \notin L$, тогда $r=0$ и $b=0$. Тогда все входы подсхемы, находящейся в области $\left.\left\{(k, l):\left(j<k \leqslant 2^{q}+1\right) \vee j<l \leqslant 2^{b}\right)\right\}$, равны 0 , и на ее выходе будет 0.

В этом случае единицы возникают на левых входах элементов с координатами $(k, i)$ при $1 \leqslant k \leqslant j$ (всего $j$ узлов) и на верхних контактах элементов $(j, l)$ при $1 \leqslant l \leqslant i$ (всего $i$ узлов). То есть потенциал схемы равен $i+j \leqslant 2^{q}+2^{b}$.

Итак, получили, что построенная схема реализует требуемую функцию и ее потенциал не превосходит $2^{q}+2^{m}+2$.

Описанные компоненты соединяются в соответствии с формулой (6), как изображено на рис. 7. Кроме непосредственно описанных блоков здесь используются их транспонированные модификации (блоки I, IV, а также транспонированная схема $\left.K_{2^{b-a} 2^{c-b}}\right)$.

Все выходы блоков, которые не используются (правые выходы блоков $Q, Q_{2}$, $K_{2^{n-c} 2^{a}}$, нижние выходы $K_{f}$, кроме последнего), могут быть удалены по лемме 10 без увеличения мощности и площади схемы.

Осталось посчитать мощность схемы. Для этого вычислим $U(K)$ и воспользуемся оценкой $\widehat{W}(K) \leqslant 2 \widehat{U}(K)$. Пусть $p=a+n-c, s=\max (a, b-a, c-b, n-c)$. Для схем $Q\left(x_{1}, \ldots, x_{k}\right)$ имеем оценку потенциала

$$
\begin{aligned}
(2 a+1)\left(2^{a}+1\right)+(2(b-a)+1)\left(2^{b-a}\right. & +1)+(2(c-b)+1)\left(2^{c-b}+1\right)+ \\
& +(2(n-c)+1)\left(2^{n-c}+1\right) \leqslant(8 s+4)\left(2^{s}+1\right) .
\end{aligned}
$$

Потенциал схемы $K_{m q}$, как уже было отмечено, не превосходит $2 m q+4 m+q$. Поэтому суммарный потенциал двух блоков $K_{2^{n-c} 2^{a}}$ и $K_{2^{b-a} 2^{c-b}}$ не превосходит

$$
2 \cdot 2^{a} \cdot 2^{n-c}+4 \cdot 2^{n-c}+2^{a}+2 \cdot 2^{b-a} \cdot 2^{c-b}+4 \cdot 2^{b-a}+2^{c-b} \leqslant 2^{p+1}+2^{n-p+1}+10 \cdot 2^{s} .
$$

Потенциал схемы $K_{f}$ не превосходит $2^{p}+2^{n-p}+2$.

Потенциал проводов, соединяющих 4 блока $Q$ с блоками $K_{m q}$ не больше, чем 4 максимальных длины провода (так как каждый дешифратор $Q$ выдает 1 лишь на одном выходе). Длина провода не больше суммы ширины $K_{m q}$ и ширины $Q$ и не превосходит $2 m+q+n$. У нас используются $K_{2^{n-c} 2^{a}}$ и $K_{2^{b-a} 2^{c-b}}^{T}$, то есть $m, q \leqslant 2^{s}$, поэтому эта величина не больше $4\left(3 \cdot 2^{s}+n\right)$

Потенциал проводов, соединяющих входы схемы со входами дешифраторов I, II, III и IV не больше произведения количества проводов произведения максимальной длины провода. Длина каждого провода не больше суммы ширины $K_{m q}$ и удвоенной 


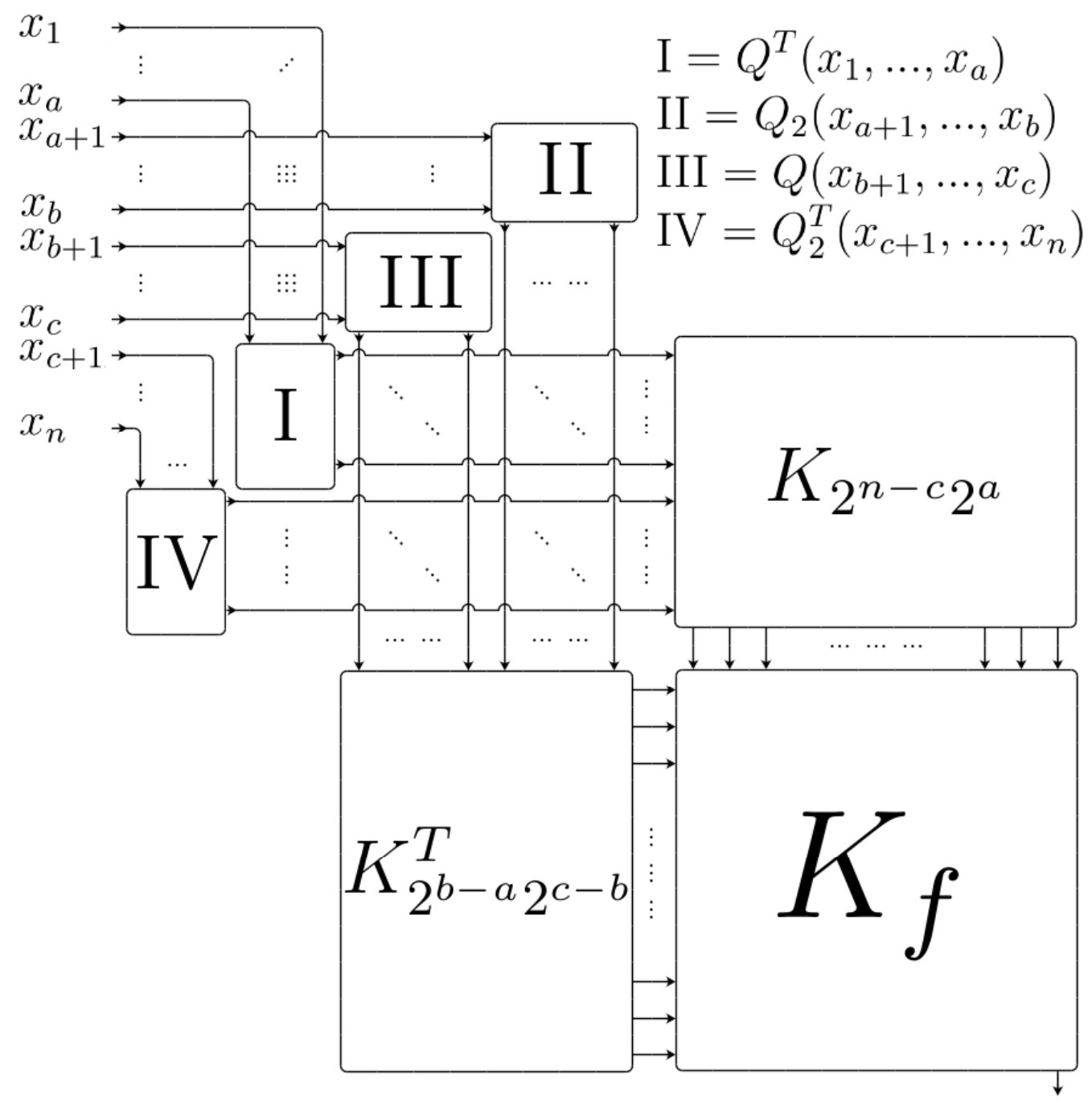

Рис. 7. Общий план схемы $K$.

ширины $Q$, то есть не больше $2 m+q+n$, поэтому потенциал не больше $n(2 m+q+n)$. Kак уже говорилось $m, q \leqslant 2^{s}$, поэтому $n(2 m+q+n) \leqslant n\left(3 \cdot 2^{s}+n\right)$.

Таким образом, суммарный потенциал всех добавленных проводов не превосходит $(n+4)\left(3 \cdot 2^{s}+n\right)$.

Теперь оценим потенциал всей схемы $K$ изображенной на рис. 7

$$
\begin{aligned}
\widehat{U}(K) \leqslant(8 s+ & 4)\left(2^{s}+1\right)+2^{p+1}+2^{n-p+1}+10 \cdot 2^{s}+2^{p}+2^{n-p}+2+ \\
& +(n+4)\left(3 \cdot 2^{s}+n\right)=3 \cdot\left(2^{p}+2^{n-p}\right)+O\left(n\left(2^{s}+n\right)\right) \text { при } n, s \rightarrow \infty .
\end{aligned}
$$

Положим $a=\left\lfloor\frac{n}{4}\right\rfloor, b=\left\lfloor\frac{n}{2}\right\rfloor, c=\left\lfloor\frac{3 n}{4}\right\rfloor$. Тогда $a, b-a, c-b, n-c \leqslant\left\lceil\frac{n}{4}\right\rceil$, значит, $s \leqslant\left\lceil\frac{n}{4}\right\rceil, p=a+n-c=\left\lfloor\frac{n}{4}\right\rfloor+\left\lceil\frac{n}{4}\right\rceil=\left\lceil\frac{n}{2}\right\rceil$. Тогда $n\left(2^{s}+n\right) \sim n 2^{n / 4}=o\left(2^{n / 2}\right)$ при $n \rightarrow \infty$. Поэтому $\widehat{U}(K) \lesssim 3\left(2^{\lfloor n / 2\rfloor}+2^{\lceil n / 2\rceil}\right)$. 
Если $n$ четно, то

$$
3\left(2^{\lfloor n / 2\rfloor}+2^{\lceil n / 2\rceil}\right)=6 \cdot 2^{n / 2} .
$$

Если $n$ нечетно, то

$$
3\left(2^{\lfloor n / 2\rfloor}+2^{\lceil n / 2\rceil}\right)=3 \cdot 2^{n / 2}\left(\sqrt{2}+\frac{1}{\sqrt{2}}\right)=\frac{9}{\sqrt{2}} 2^{n / 2} .
$$

Итак, в любом случае $\widehat{U}(K) \lesssim \frac{9}{\sqrt{2}} 2^{\lceil n / 2\rceil}$, отсюда $\widehat{W}(K) \leqslant 2 \widehat{U}(K) \lesssim 9 \sqrt{2} \cdot 2^{n / 2}$.

Длина полученной схемы равна $n-c+a+2 \cdot 2^{b-a}+2^{c-b}+2^{a} 2^{n-c}+1 \leqslant 2^{p}+p+1+3 \cdot 2^{s}$. Ширина схемы равна $c+2 \cdot 2^{n-c}+2^{a}+2^{b-a} 2^{c-b} \leqslant 2^{n-p}+c+3 \cdot 2^{s}$. Таким образом, ее площадь не превосходит

$\left(2^{p}+p+1+3 \cdot 2^{s}\right)\left(2^{n-p}+c+3 \cdot 2^{s}\right)=2^{\left\lceil\frac{n}{2}\right\rceil}(1+o(1)) 2^{\left\lfloor\frac{n}{2}\right\rfloor}(1+o(1))=2^{n}(1+o(1))$ при $n \rightarrow \infty$.

Отсюда, в частности видно, что верхняя оценка функции Шеннона для всех мер мощности схем, реализующих булевы функции от $n$ переменных, имеет порядок $2^{\frac{n}{2}}$.

\section{5. Нижняя оценка мощности}

Скажем, что в схеме $K$ с входами $X_{1}, \ldots, X_{n}$ на множестве узлов $\left\{R_{1}, \ldots, R_{m}\right\}$ peaлизуется булев оператор $F=\left(f_{1}\left(x_{1}, \ldots, x_{n}\right), \ldots, f_{m}\left(x_{1}, \ldots, x_{n}\right)\right)$, если при подаче на вход $X_{i}$ переменной $x_{i}, i=1, \ldots, n$, в узле $R_{j}$ реализуется $f_{j}\left(x_{1}, \ldots, x_{n}\right), j=1, \ldots, m$.

Для доказательства нижней оценки для средней мощности понадобятся следующие леммы.

Лемма 12. Если в схеме $K$ с входами $X_{1}, \ldots, X_{n}$ на множестве узлов $\left\{R_{1}, \ldots, R_{m}\right\}$ реализуется булев оператор $F=\left(f_{1}(x), \ldots, f_{m}(x)\right)$ и $F(x) \neq F(y)$, то

$$
w_{K}(x, y) \geqslant \max _{j: f_{j}(x) \neq f_{j}(y)} \min _{i: x_{i} \neq y_{i}} \rho_{K}\left(X_{i}, R_{j}\right) .
$$

Доказательство. Зафиксируем произвольные $x$ и $y$, для которых $F(x) \neq F(y)$. Обозначим $I=\left\{X_{i}: x_{i} \neq y_{i}\right\}, l:=\max _{j: f_{j}(x) \neq f_{j}(y)} \min _{i: x_{i} \neq y_{i}} \rho_{K}\left(X_{i}, R_{j}\right)$.

Пусть максимум по $j$ достигается на выходе $R_{j_{0}}$. Этот выход подключен к некоторому элементу $e_{0}$. Обозначим через $K_{0}=\left\{e_{0}\right\}$ подсхему $K$ с выходом $R_{j_{0}}$. Будем строить последовательность подсхем $K_{i}$ по индукции.

Пусть $K_{i}$ является подсхемой $K$, причем выход $R_{j_{0}}$ является выходом $K_{i}$. При смене входов схемы $K$ с $x$ на $y$ меняется значение на выходе $R_{j_{0}}$ подсхемы $K_{i}$, a, значит, должно было поменяться значение на одном из входов $K_{i}$, причем выход $R_{j_{0}}$ зависит от этого входа в рамках схемы $K_{i}$. Есть 2 варианта.

- Этот вход является выходом некоторого элемента $e_{i+1}$; тогда положим $K_{i+1}=$ $K_{i} \cup\left\{e_{i+1}\right\}$.

- Этот вход является входом схемы $K$; на этом построение завершается.

В схеме $K$ конечное число элементов, поэтому на некотором шаге построения последовательности возникнет второй случай. 
Тогда тот вход последнего добавленного элемента, на котором поменялось значение, является входом схемы $K_{i}$, а, значит, должен принадлежать $I$, поскольку $x$ отличается от $y$ только компонентами из $I$.

Схеме $K_{i}$ соответствует граф, одной из вершин которого является вход $X_{i_{0}} \in I$, а другой - выход $R_{j_{0}}$. Причем выход зависит от входа в рамках схемы $K_{i}$, а, значит, существует цепочка в графе схемы от входа $X_{i_{0}}$ к выходу $R_{j_{0}}$. Число ребер в этой цепочке не менее

$$
\min _{X_{i} \in I} \rho_{K}\left(X_{i}, R_{j_{0}}\right)=\min _{i: x_{i} \neq y_{i}} \rho_{K}\left(X_{i}, R_{j_{0}}\right)=\max _{j: f_{j}(x) \neq f_{j}(y)} \min _{i: x_{i} \neq y_{i}} \rho_{K}\left(X_{i}, R_{j}\right)=l .
$$

Каждому ребру в цепочке от входа к выходу соответствует некоторый элемент схемы, причем элементы не повторяются, иначе был бы ориентированный цикл. Значит, число элементов в схеме не меньше, чем длина цепочки ребер от входа к выходу, то есть $\left|K_{i}\right| \geqslant l$. По построению на одном из выходов каждого элемента подсхемы $K_{i}$ поменялось значение, значит, $w_{K}(x, y) \geqslant\left|K_{i}\right| \geqslant l$.

Пусть

$X_{i}(f):=\left\{x \in\{0,1\}^{n-1}: f\left(x_{1}, \ldots, x_{i-1}, 0, x_{i}, \ldots, x_{n-1}\right) \neq f\left(x_{1}, \ldots, x_{i-1}, 1, x_{i}, \ldots, x_{n-1}\right)\right\}$,

$l_{i}$ - расстояние между $i$-м входом и выходом схемы. По сути $X_{i}$ - это множество наборов переменных кроме $i$-й, на которых функция $f$ не зависит существенно от $i$-й переменной.

Лемма 13. Если схема $K$ реализует булеву функиию $f\left(x_{1}, \ldots, x_{n}\right)$, то для всех $i=1, \ldots, n$

$$
W(K) \geqslant \frac{\left|X_{i}(f)\right| l_{i}}{2^{n}}
$$

Доказательство. Без ограничения общности можно считать, что $i=1$. Если $x \in$ $X_{1}$, то $f(0, x) \neq f(1, x)$, и по лемме 12 получим, что $w_{K}((0, x),(1, x)) \geqslant l_{1}$, поскольку выход у схемы один, а $(0, x)$ и $(1, x)$ отличаются только первой компонентой. По неравенству треугольника $(2) w_{K}((0, x),(1, x)) \leqslant w_{K}((0, x), y)+w_{K}((1, x), y)$. Отсюда

$$
\begin{array}{r}
W(K)=\sum_{y, z \in\{0,1\}^{n}} \frac{w_{K}(z, y)}{2^{2 n}} \geqslant \sum_{x \in X_{1}} \sum_{y \in\{0,1\}^{n}} \frac{1}{2^{2 n}}(\underbrace{w_{K}((0, x), y)+w_{K}((1, x), y)}_{\geqslant l_{1}}) \geqslant \\
\geqslant\left|X_{1}(f)\right| \frac{1}{2^{2 n}} 2^{n} l_{1}=\frac{\left|X_{1}(f)\right| l_{1}}{2^{n}} .
\end{array}
$$

Лемма 14. Если $n \rightarrow \infty u \log _{2} m=o\left(2^{n}\right)$, то доля булевых операторов $f$ с $n$ входами и $т$ выходами, для которых $W(f) \leqslant C \sqrt{m} 2^{n / 2}$ u $C=\frac{1}{\sqrt{1000 \log _{2}|E|}}$, стремится к нулю.

Доказательство. Зафиксируем некоторое число $\varepsilon>0$. Пусть $F_{\varepsilon}-$ множество таких операторов $f=\left(f_{1}, \ldots, f_{m}\right)$ с $n$ входами и $m$ выходами, что $\left|X_{i}\left(f_{j}\right)\right| \geqslant 2^{n-2-\varepsilon}$ для всех $i=1, \ldots, n, j=1, \ldots, m$. То есть $F_{\varepsilon}-$ множество таких операторов, у которых каждый выход существенно зависит от каждой переменной на не менее чем $2^{n-2-\varepsilon}$ 
наборах. Посчитаем количество элементов в $F_{\varepsilon}$

$$
\begin{aligned}
\left|F_{\varepsilon}\right| & =2^{m 2^{n}}-\left|\bigcup_{i, j}\left\{f:\left|X_{i}\left(f_{j}\right)\right|<2^{n-2-\varepsilon}\right\}\right| \geqslant \\
& \geqslant 2^{m 2^{n}}-\sum_{j=1}^{m} \sum_{i=1}^{n}\left|\left\{f:\left|X_{i}\left(f_{j}\right)\right|<2^{n-2-\varepsilon}\right\}\right|= \\
& =2^{m 2^{n}}-\sum_{j=1}^{m} \sum_{i=1}^{n} 2^{(m-1) 2^{n}}\left|\left\{f_{j}:\left|X_{i}\left(f_{j}\right)\right|<2^{n-2-\varepsilon}\right\}\right|= \\
& =2^{(m-1) 2^{n}}\left(2^{2^{n}}-m n\left|\left\{f_{1}:\left|X_{1}\left(f_{1}\right)\right|<2^{n-2-\varepsilon}\right\}\right|\right) .
\end{aligned}
$$

Посчитаем $n_{t}=\left|\left\{f_{1}:\left|X_{1}\left(f_{1}\right)\right|=t\right\}\right|$. Заметим, что функция $f_{1}$ однозначно задается значениями $g(x)=f_{1}(0, x), x \in\{0,1\}^{n-1}$ и множеством $X_{1}=X_{1}\left(f_{1}\right) \subseteq\{0,1\}^{n-1}$, а именно,

$$
f_{1}\left(x_{1}, x\right)= \begin{cases}g(x), & x_{1}=0 \vee x \notin X_{1} \\ \overline{g(x)}, & x_{1}=1 \wedge x \in X_{1} .\end{cases}
$$

Есть $2^{2^{n-1}}$ различных функций $g(x), x \in\{0,1\}^{n-1}$, и $C_{2^{2^{n-1}}}^{t}$ различных множеств $X_{1} \subseteq\{0,1\}^{n-1}$ мощности $t$. Поэтому $n_{t}=2^{2^{n-1}} C_{2^{n-1}}^{t}$ и

$$
\left|\left\{f_{1}:\left|X_{1}\left(f_{1}\right)\right|<2^{n-2-\varepsilon}\right\}\right|<\sum_{t=0}^{\left\lfloor 2^{n-2-\varepsilon}\right\rfloor} n_{t}=2^{2^{n-1}} \sum_{t=0}^{\left\lfloor 2^{n-2-\varepsilon}\right\rfloor} C_{2^{n-1}}^{t} \leqslant 2^{2^{n-1}} \cdot 2^{2^{n-1} H\left(\frac{1}{2^{1+\varepsilon}}\right)},
$$

где $H(x)=-x \log _{2} x-(1-x) \log _{2}(1-x)-$ функция энтропии $^{1}$.

Обозначим $d(\varepsilon)=1-H\left(\frac{1}{2^{1+\varepsilon}}\right)$. Тогда $\left|\left\{f_{1}:\left|X_{1}\left(f_{1}\right)\right|<2^{n-2-\varepsilon}\right\}\right| \leqslant 2^{2^{n-1}(2-d(\varepsilon))}$. Вспомним, что функция $H$ строго выпукла вверх, имеет максимум при $x=\frac{1}{2}$, причем $H\left(\frac{1}{2}\right)=1$. Поэтому $H\left(\frac{1}{2^{1+\varepsilon}}\right)<1$, а, значит, $d(\varepsilon)>0$. Отсюда следует, что

$\left|F_{\varepsilon}\right| \geqslant 2^{(m-1) 2^{n}}\left(2^{2^{n}}-m n 2^{2^{n-1}(2-d(\varepsilon))}\right)=2^{m 2^{n}}\left(1-n 2^{o\left(2^{n}\right)} 2^{-d(\varepsilon) 2^{n-1}}\right) \sim 2^{m 2^{n}}$ при $n \rightarrow \infty$.

Здесь мы учли, что $m=2^{o\left(2^{n}\right)}$.

Зафиксируем некоторое число $w_{0} \geqslant 1$. Рассмотрим множество схем $K$, реализующих операторы из $F_{\varepsilon}$, для которых $W(K) \leqslant w_{0}$. Идея состоит в том, чтобы найти свойства, которыми обладают все операторы, реализуемые такими схемами. Потом мы оценим сверху количество операторов, обладающих этими свойствами и получим нужную оценку. Пусть $L\left(w_{0}\right)$ - множество таких операторов $f \in F_{\varepsilon}$, которые можно реализовать схемами мощностью не более $w_{0}$.

Рассмотрим такую схему $K$. Пусть $l$ - максимальное расстояние от входа до выхода в схеме $K$. Зафиксируем выход $Y$, на котором этот максимум достигается; на этом выходе реализуется некоторая функция $f_{j}$. Поскольку схема $K$ реализует оператор из $F_{\varepsilon}$, то, применяя лемму 13 к схеме $K$ и функции $f_{j}$, получаем

$$
w_{0} \geqslant W(K) \geqslant \max _{i=1 \ldots n} \frac{\left|X_{i}\left(f_{j}\right)\right| l_{i}}{2^{n}} \geqslant \frac{2^{n-2-\varepsilon}}{2^{n}} l=\frac{l}{2^{2+\varepsilon}} .
$$

\footnotetext{
1 Здесь мы воспользовались тем фактом, что $\sum_{k=0}^{s} C_{n}^{k} \leqslant 2^{n H(s / n)}$ при $s \leqslant n / 2$, доказательство этого можно прочитать, например, в [6, стр.32, теорема 2.9].
} 
Отсюда следует, что $l \leqslant 2^{2+\varepsilon} w_{0}$. Зафиксируем некоторое число $r \geqslant 2^{2+\varepsilon} w_{0}$. Обозначим через $K_{r}$ множество элементов схемы $K$, расстояние от входов которых до одного из входов схемы не более $r$. Выходы схемы $K$ отстоят от входов не больше, чем на $l \leqslant r$, то есть являются выходами $K_{r}$. Входы всех этих элементов по неравенству треугольника отстоят от выхода $Y$ не более, чем на $l$, а, значит, сами элементы отстоят от выхода $Y$ не более, чем на $l+r$ по манхеттеновской метрике. Поэтому их не больше, чем число элементов в шаре радиуса $r+l$, то есть

$$
V(r+l):=1+\sum_{i=1}^{l+r} 4 i=1+2(l+r)(l+r+1) .
$$

Оставшуюся часть схемы обозначим через $\bar{K}_{r}$. Поскольку среди входов схемы $\bar{K}_{r}$ нет входов схемы $K$, то они все являются выходами $K_{r}$. Пусть на них реализуется оператор $G$ (так же как на множестве узлов схемы $K)$. Пусть $M_{r}=\max _{g \in \operatorname{Im} G}\left|G^{-1}(g)\right|$. Тогда

$$
P\{G(x)=G(y)\}=\sum_{x \in\{0,1\}^{n}} P(x) P\left(G^{-1}(G(x))\right) \leqslant \frac{M_{r}}{2^{n}} .
$$

По лемме 12 если $G(x) \neq G(y)$, то $w_{K}(x, y) \geqslant r$. Поэтому $W(K) \geqslant$ $P\{G(x) \neq G(y)\} r \geqslant \frac{2^{n}-M_{r}}{2^{n}} r$. Отсюда $M_{r} \geqslant 2^{n}\left(1-\frac{w_{0}}{r}\right)$. Пусть $M_{r}=\left|G^{-1}\left(g_{0}\right)\right|$. Обозначим $D=G^{-1}\left(g_{0}\right)$. Тогда для всех $x \in D$ на входах $\bar{K}_{r}$ будет константный вход $g_{0}$, a, значит, и на выходах также будет некоторый константный выход $h_{0}$. Рассмотрим схему $K(r)$, которую можно получить из $K_{r}$, если подать на входы со стороны $\bar{K}_{r}$ значение $h_{0}$ и «протащить» его через все элементы, то есть если константа $c$ подана на $i$-й вход элемента, реализующего функцию $a\left(x_{1}, \ldots, x_{m}\right)$, то заменяем его на элемент, реализующий функцию $\tilde{a}\left(x_{1}, \ldots, x_{i-1}, x_{i+1}, \ldots, x_{n}\right)=a\left(x_{1}, \ldots, x_{i-1}, c, x_{i+1}, \ldots, x_{n}\right)$. Таким образом, схема $K(r)$ имеет площадь не более $\left|K_{r}\right| \leqslant V(r+l)$ и реализует оператор, совпадающий с $f$ на множестве $D$, то есть не менее, чем на $M_{r}$ наборах.

Значит, для любого $f \in L\left(w_{0}\right)$ существует схема, помещающаяся в круге радиуса $r+l$ (далее будем говорить, что радиус схемы не более $r+l$ ) и реализующая оператор $f^{\prime}$, совпадающий с $f$ не менее, чем на $M_{r}$ наборах. Причем при некотором $\varepsilon>0$

$$
l \leqslant 2^{2+\varepsilon} w_{0}, \quad r \geqslant 2^{2+\varepsilon} w_{0}, \quad M_{r} \geqslant 2^{n}\left(1-\frac{w_{0}}{r}\right) .
$$

Пусть $N\left(w_{0}\right)$ - количество операторов с таким свойством. Тогда $\left|L\left(w_{0}\right)\right| \leqslant N\left(w_{0}\right)$. Оценим $N\left(w_{0}\right)$ в два этапа.

(1) Сначала найдём количество схем радиуса не более $r+l$. Каждую такую схему $K: G \rightarrow E$ можно взаимно однозначно доопределить до схемы на множестве $B(O, r+l), K_{0}(g)=\left\{\begin{array}{ll}K(g), & g \in G, \\ \lambda & g \notin G,\end{array}\right.$ где $B(0, r+l)$ - круг с центром $O$ (выход схемы) радиуса $r+l$ в манхеттеновской метрике. То есть мы заполняем свободные клетки пустыми элементами. Количество схем не больше, чем количество отображений из $B(O, r+l)$ в $E$, которое равно $|E|^{V(r+l)}$.

(2) Возьмем произвольную такую схему. Она реализует некоторый оператор $g$ и все $n ! m !$ операторов, получающиеся из него перестановкой входов и выходов. Оценим число операторов, совпадающих с $g$ не менее, чем на $M_{r}$ наборах. 
Любому такому оператору однозначно сопоставляется множество наборов $D$, на которых он совпадает с $g$, и доопределение на остальных наборах

$$
f_{j}(x)= \begin{cases}\frac{g_{j}(x),}{g_{j}(x),}, & x \notin D_{j}, \\ x \notin D_{j} .\end{cases}
$$

Поэтому с точностью до перестановки входов и выходов операторов не больше, чем способов выбрать область $D \subseteq\{0,1\}^{n}:|D|=M_{r}$ и доопределить на оставшихся $2^{n}-M_{r}$ наборах. Есть $C_{2^{n}}^{M_{r}}$ способов выбрать $D \subseteq\{0,1\}^{n}:|D|=$ $M_{r}$ и $2^{m\left(2^{n}-M_{r}\right)}$ способов доопределения. Поэтому всего операторов не более, чем

$$
m ! n ! C_{2^{n}}^{M_{r}} 2^{m\left(2^{n}-M_{r}\right)} \leqslant m ! n ! 2^{2^{n} H\left(\frac{M_{r}}{2^{n}}\right)+m\left(2^{n}-M_{r}\right)} \leqslant m ! n ! 2^{2^{n}\left(H\left(\frac{w_{0}}{r}\right)+m \frac{w_{0}}{r}\right) .} .
$$

Здесь мы учли, что $\frac{M_{r}}{2^{n}} \geqslant 1-\frac{w_{0}}{r}>\frac{1}{2}$ согласно (7). Учитывая, что функция энтропии $H(x)$ убывает при $x \geqslant \frac{1}{2}$ и $H(x)=H(1-x)$, находим: $H\left(\frac{M_{r}}{2^{n}}\right) \leqslant$ $H\left(1-\frac{w_{0}}{r}\right)=H\left(\frac{w_{0}}{r}\right)$.

Тогда, учитывая $(7)$ и обозначая $h(x)=m x+H(x)$, получим

$$
N\left(w_{0}\right) \leqslant|E|^{V(r+l)} m ! n ! 2^{2^{n} h\left(\frac{w_{0}}{r}\right)} \leqslant n^{n} m^{m}|E|^{V\left(2^{2+\varepsilon} w_{0}+r\right)} 2^{2^{n} h\left(\frac{w_{0}}{r}\right)} .
$$

Отсюда следует, что

$$
\log _{2} N\left(w_{0}\right) \leqslant n \log _{2} n+m \log _{2} m+V\left(2^{2+\varepsilon} w_{0}+r\right) \log _{2}|E|+2^{n} h\left(\frac{w_{0}}{r}\right) .
$$

Полагая $r=7 w_{0}, \varepsilon=\log _{2}(\sqrt{122}-7)-2$ и учитывая, что $V(x)=1+2 x(x+1) \leqslant$ $2(x+1)^{2}$, получаем

$$
\begin{gathered}
\log _{2} N\left(w_{0}\right) \leqslant n \log _{2} n+m \log _{2} m+2 \log _{2}|E|\left(\sqrt{122} w_{0}+1\right)^{2}+2^{n} h(1 / 7)< \\
<244\left(w_{0}+1\right)^{2} \log _{2}|E|+\left(\frac{3}{4}+\frac{m-1}{7}\right) 2^{n}+n \log _{2} n+m \log _{2} m .
\end{gathered}
$$

Если $w_{0} \leqslant C \sqrt{m} 2^{n / 2}$, где $C=\frac{1}{\sqrt{1000 \log _{2}|E|}}$, то

$$
\begin{aligned}
\log _{2} N\left(C \sqrt{m} 2^{n / 2}\right) & <2^{n}\left(m \frac{244 \log _{2}|E|}{1000 \log _{2}|E|}+\frac{3}{4}+\frac{m-1}{7}\right)+n \log _{2} n+m \log _{2} m \leqslant \\
\leqslant & 2^{n}\left(1-\frac{3}{500}+\frac{m-1}{2}\right)+n \log _{2} n+m \log _{2} m .
\end{aligned}
$$

Отсюда

$$
\begin{aligned}
& \frac{N\left(C \sqrt{m} 2^{n / 2}\right)}{2^{m 2^{n}}}<\frac{2^{2^{n}\left(1-\frac{3}{500}+\frac{m-1}{2}+\frac{n \log _{2} n+m \log _{2} m}{m 2^{n}}\right)}}{2^{m 2^{n}}}=\frac{1}{2^{2^{n} \cdot\left(\frac{3}{500}+\frac{m-1}{2}-\frac{m \log _{2} m+n \log _{2} n}{2^{n}}\right)}}= \\
& =\frac{1}{2^{2^{n} \cdot\left(\frac{3}{500}+\frac{m-1}{2}+o(m)\right)} \rightarrow 0 \text { при } n \rightarrow \infty .}
\end{aligned}
$$

Как следствие лемм 11 и 14 при $m=1$ получаем результат утверждение 2. 


\section{Список литературы}

1. Кравцов С. С., О реализации функций алгебры логики в одном классе схем из функциональных и коммутационных элементов. Проблемы кибернетики (1967) 19, 285-293.

2. Жуков Д. А., О вычислении частичных булевых функций клеточными схемами. Дискретный анализ и исследование операщий (2004) 11, №1, 32-40.

3. Шкаликова Н. А., О реализации булевых функций схемами из клеточных элементов. Математические вопросы кибернетики (1989) 2, 177-197.

4. Касим-Заде О. М., Об одной мере активности схем из функциональных элементов. Математические вопросы кибернетики (1992) 4, 218-228.

5. Шуткин Ю. С., Асимптотически оптимальная реализация булевых функций информационными графами. Дискретная математика (2011) 23, №4, 80-102.

6. Чашкин А.В., Лекиии по дискретной математике. МГУ Мехмат, Москва, 2007.

Статья поступила 11.10.2013. 